\title{
Laminar- and Target-Specific Amygdalar Inputs in Rat Primary Gustatory Cortex
}

\author{
(D) Melissa S. Haley, Alfredo Fontanini, ${ }^{*}$ and ${ }^{\top}$ Arianna Maffei* \\ Department of Neurobiology and Behavior and Program in Neuroscience, State University of New York-Stony Brook, Stony Brook, New York 11794
}

\begin{abstract}
The primary gustatory cortex $(\mathrm{GC})$ receives projections from the basolateral nucleus of the amygdala (BLA). Behavioral and electrophysiological studies demonstrated that this projection is involved in encoding the hedonic value of taste and is a source of anticipatory activity in GC. Anatomically, this projection is largest in the agranular portion of GC; however, its synaptic targets and synaptic properties are currently unknown. In vivo electrophysiological recordings report conflicting evidence about BLA afferents either selectively activating excitatory neurons or driving a compound response consistent with the activation of inhibitory circuits. Here we demonstrate that BLA afferents directly activate excitatory neurons and two distinct populations of inhibitory neurons in both superficial and deep layers of rat GC. BLA afferents recruit different proportions of excitatory and inhibitory neurons and show distinct patterns of circuit activation in the superficial and deep layers of GC. These results provide the first circuit-level analysis of BLA inputs to a sensory area. Laminar-and target-specific differences of BLA inputs likely explain the complexity of amygdalocortical interactions during sensory processing.
\end{abstract}

Key words: basolateral amygdala; excitation; gustatory cortex; inhibition; microcircuitry; optogenetics

\section{Significance Statement}

Projections from the basolateral nucleus of the amygdala (BLA) to the cortex convey information about the emotional value and the expectation of a sensory stimulus. Although much work has been done to establish the behavioral role of BLA inputs to sensory cortices, very little is known about the circuit organization of BLA projections. Here we provide the first in-depth analysis of connectivity and synaptic properties of the BLA input to the gustatory cortex. We show that BLA afferents activate excitatory and inhibitory circuits in a layer-specific and pattern-specific manner. Our results provide important new information about how neural circuits establishing the hedonic value of sensory stimuli and driving anticipatory behaviors are organized at the synaptic level.

\section{Introduction}

The basolateral nucleus of the amygdala (BLA) plays a crucial role in a multitude of functions, including reward processing, emotional learning, attention, and anticipation of outcomes on the basis of predictive cues (Baxter and Murray, 2002; Phelps and LeDouz, 2005; Balleine and Killcross, 2006; Pessoa and Adolphs, 2010). BLA is also heavily involved in modulating sensory processes (Ferreira et al., 2005; Fontanini and Katz, 2006; Grossman

\footnotetext{
Received Aug. 27, 2015; revised Dec. 15, 2015; accepted Jan. 7, 2016.

Author contributions: M.S.H., A.F., and A.M. designed research; M.S.H. performed research; M.S.H. analyzed data; M.S.H., A.F., and A.M. wrote the paper.

This work was supported by National Institutes of Health DC01770 to A.F. and A.M. We thank Martha Stone, Olivia Swanson, and Drs. Roberta Tatti, Trevor Griffen, Michelle Kloc, Yury Garkun, Lang Wang, Matthew Gardner, Chad Samuelsen, Haixin Liu, Ahmad Jezzini, and Roberto Vincis.

The authors declare no competing financial interests.

${ }^{*}$ A.F. and A.M. contributed equally to this work.

Correspondence should be addressed to either Dr. Alfredo Fontanini or Dr. Arianna Maffei, Department of Neurobiology and Behavior, Life Science Building, Room 548, State University of New York-Stony Brook, Stony Brook, NY 11794, E-mail: Alfredo.fontanini@stonybrook.edu or Arianna.maffei@stonybrook.edu.

DOI:10.1523/JNEUROSCI.3224-15.2016

Copyright $\odot 2016$ the authors $\quad 0270-6474 / 16 / 362623-15 \$ 15.00 / 0$
}

et al., 2008; Chavez et al., 2009; Fontanini et al., 2009; Roesch et al., 2010; Rodríguez-Durán et al., 2011). This function has been extensively studied in reference to gustatory processing due to the dense connection between BLA and the gustatory cortex (GC) (Saper, 1982; Allen et al., 1991; Maffei et al., 2012).

Experiments combining pharmacological inactivation with ensemble recordings in GC showed that amygdalar inputs are necessary for coding taste palatability (Piette et al., 2012) and for the processing of anticipatory cues (Samuelsen et al., 2012, 2013; Gardner and Fontanini, 2014). Silencing BLA greatly affects the firing rates of GC neurons in response to taste stimulation, specifically suppresses palatability processing (Piette et al., 2012), and reduces the magnitude of spiking responses to taste-predicting cues in GC (Samuelsen et al., 2012). Additional electrophysiological, behavioral, and pharmacological evidence points at the importance of this connection for changes in palatability occurring with taste learning (Ferreira et al., 2005; Grossman et al., 2008; Rodríguez-Durán et al., 2011).

Although the functional roles of the BLA-GC projection have been extensively studied, much less is known about the synaptic 
organization and properties of this input. Anatomical data indicate that BLA afferents target deep and superficial layers in agranular GC (aGC) and part of dysgranular GC (dGC) (Saper, 1982; Allen et al., 1991). Initial reports suggested that BLA afferents synapse onto pyramidal neurons (Smith and Paré, 1994; Paré et al., 1995). Other work, however, showed that stimulation of BLA can result in a combination of excitatory and inhibitory effects (Yamamoto et al., 1984; Hanamori, 2009; Stone et al., 2011). For instance, a recent study showed that a single electrical stimulus in BLA evokes a compound synaptic response consistent with activation of excitatory and inhibitory neurons in GC (Stone et al., 2011). It is unknown whether these responses result from the recruitment of feedforward inhibition or are polysynaptic. Furthermore, as most of the physiological studies on the BLA-GC connection have not taken into account the laminar distribution of the responses, no information is available on the possible differential action of this input on different cortical layers.

Here we show that BLA inputs activate feedforward excitatory and inhibitory circuits in superficial and deep layers of aGC. BLA afferents make monosynaptic connections with pyramidal neurons and two distinct populations of inhibitory neurons. The connectivity of this projection shows layer-specific differences. BLA axons contact a large percentage of excitatory and inhibitory neurons in the superficial layers, but the connectivity is reduced in the deep layers. In addition, while the strength of BLA inputs onto inhibitory neurons is similar across aGC, evoked synaptic responses onto pyramidal neurons are much larger in the superficial layers. Analysis of excitatory and inhibitory synaptic currents evoked onto pyramidal neurons using different activity regimens unveiled differences in the mode of activation of the circuits in the superficial and deep layers. Our results provide the first report of the synaptic organization of BLA inputs in aGC and highlight the complexity of amygdalocortical interactions under distinct activity regimens.

\section{Materials and Methods}

All surgical and experimental procedures were approved by the Institutional Animal Care and Use Committee of Stony Brook University and followed the guidelines of the National Institutes of Health.

Surgical procedures. We used an optogenetic approach to examine the BLA-GC synapse in an acute slice preparation. Briefly, Long-Evans rats of both sexes, aged $14 \mathrm{~d}$ (P14), were anesthetized with a mixture containing $70 \mathrm{mg} / \mathrm{kg}$ ketamine, $0.7 \mathrm{mg} / \mathrm{kg}$ acepromazine, and $3.5 \mathrm{mg} / \mathrm{kg}$ xylazine. Adeno-associated viral particles, serotype 9, that drive the expression of Channelrhodopsin2 (ChR2) and enhanced yellow fluorescent protein under the CAG promoter (AAV9-ChR2-EYFP) (Petreanu et al., 2007; Wang et al., 2013) were injected into the BLA (2.1 $\mathrm{mm}$ posterior to bregma; $4.7 \mathrm{~mm}$ lateral to midline; $7.0 \mathrm{~mm}$ below the pia) using a nanoject pressure injector (Drummond Nanoject II; 500 nl volume containing $50 \times 10^{12}$ particles/nl). AAV9 was chosen because it is primarily transported anterogradely. Furthermore, ChR2 expressed via AAV9 does not alter the short-term dynamics of evoked responses (Jackman et al., 2014). The lack of any backfilled somata in GC in all of our preparations further confirms the forward direction of transport of our construct and indicates that only BLA terminal fields in GC were activated by light pulses. To verify injection coordinates, sections containing BLA were imaged with confocal microscopy (Olympus Fluoview). Coronal slices containing the primary gustatory cortex (1.5 $\mathrm{mm}$ to bregma) were prepared $14 \mathrm{~d}$ after the injection, and the level of viral expression in GC was quantified by analyzing the intensity of EYFP expression across all lamina.

To ensure consistency of ChR2-EYFP expression across preparations, a calibration curve of the EYFP fluorescence intensity was obtained across several slices from several rats (Wang et al., 2013). The rationale for using a calibration curve in this context is to assess the degree of variability of the expression of the construct across preparations and include in the analysis only data obtained from preparations with comparable levels of the light-gated conductance. This information is critical to ensure that possible differences in the dataset are not biased by differences in the level of expression of the viral construct. In our preparation, BLA neurons are not preserved, therefore making sure that synaptic data are obtained from preparations in which a comparable proportion of terminal fields is activated is key to ensure that possible differences in the synaptic data are due to actual differences in synaptic properties. As EYFP is coexpressed with ChR2, measuring the level of EYFP fluorescence in GC is a good approximation of the level of expression of ChR2 in the terminal fields. The calibration curve shown in Figure $3 A$ was obtained from several preparations ( $n=10$ rats) used specifically to assess histologically the level of expression of the AAV9-ChR2-EYFP construct in our preparation. Two weeks after injection, animals were perfused intracardially with $4 \%$ PFA. Thin slices $(50 \mu \mathrm{m})$ containing the BLA and aGC were prepared, counterstained with Hoechst to visualize layers, mounted, and coverslipped. Images of BLA and aGC were taken using a confocal microscope and analyzed offline with ImageJ. In these 10 animals, we verified the goodness of our stereotaxic coordinates by assessing that the injection site was specific to BLA with no aspecific expression or leakage of the construct in the areas surrounding BLA. We then quantified the intensity of EYFP in aGC, as a measure of the level of coexpression of ChR2, over a 100- $\mu \mathrm{m}$-wide region of interest spanning aGC from the pia to the claustrum. The fluorescence intensity was averaged across at least 3 slices for each of the 10 animals and then across animals. The SD across preparations was also determined. These data were used to establish the calibration curve (see Fig. 3A). At the end of each electrophysiological experiment, slices were fixed to assess the identity and location of recorded neurons and to quantify the intensity of EYFP fluorescence. The level of EYFP fluorescence was compared with the calibration curve, and only recordings from preparations whose EYFP expression was within 1 $\mathrm{SD}$ of the calibration curve were included in the analysis. After establishing the calibration curve, the profile of EYFP expression was quantified for each slice used for electrophysiological recordings, and only data obtained from slices whose expression profile was within 1 SD from the calibration curve were included in the analysis (see Fig. $3 A$ ). This allowed for comparisons across preparations with similar levels of ChR2 expression in the BLA terminal fields in GC.

Electrophysiology. Following a minimum incubation period of $14 \mathrm{~d}$ after surgery, rats were anesthetized with isoflurane and acute coronal slices containing GC were prepared. Briefly, once anesthetized, rats were decapitated and the brain was dissected out in ice-cold, oxygenated ACSF. Slices $(300 \mu \mathrm{m})$ were cut with a vibratome (Leica VT1000) and allowed to recover in a continuously oxygenated chamber at room temperature. To verify the specificity of the injection site, coronal slices containing BLA were also prepared from each brain. In addition, to ensure sufficient levels of expression of the construct, patch-clamp recordings were performed in BLA at the start of each experiment (see Fig. 1). After visual and functional confirmation of successful BLA injections, patch-clamp recordings were obtained from visually identified neurons in the supragranular (sGC) and infragranular (iGC) laminae of aGC. Under DIC optics, aGC was identified from its proximity to piriform cortex and the rhinal fissure, and the absence of layer 4 .

Pyramidal neurons, fast-spiking, parvalbumin-expressing neurons (FS-PV), and regular-spiking, nonpyramidal, somatostatin-expressing neurons (RSNP-SOM) were identified online by their firing patterns in response to hyperpolarizing and depolarizing current steps $(50 \mathrm{pA})$ delivered to the cell in current-clamp mode. Their identity was confirmed by post hoc morphology reconstruction and immunostaining with specific markers.

BLA terminal fields (or BLA neurons in slices containing BLA) were activated with brief light pulses (5 ms) delivered through a $40 \times$ waterimmersion objective using a blue LED ( $470 \mathrm{~nm}$, ThorLabs) mounted on the fluorescence pathway of an upright microscope (Olympus BX51WI). LED stimulation intensity, duration, and frequency were controlled through an LED driver (ThorLabs) connected to the digital output of a Multipatch clamp amplifier (HEKA). The light power at the level of the tissue was quantified with an optical power meter (Coherent). In a subset 
of experiments designed to isolate excitatory and inhibitory currents evoked by BLA terminal field stimulation, recordings were made using a cesium-based internal solution (see solutions). To isolate combined AMPA-NMDA currents, neurons were held at the $\mathrm{E}_{\text {rev }}$ for $\mathrm{GABA}_{\mathrm{A}^{-}}$ mediated currents, whereas $\mathrm{GABA}_{\mathrm{A}}$-mediated currents were isolated by holding neurons at the $\mathrm{E}_{\mathrm{rev}}$ for excitatory currents. The $\mathrm{E}_{\mathrm{rev}}$ for each was determined by holding neurons between $-50 \mathrm{mV}$ and $-30 \mathrm{mV}$ in $5 \mathrm{mV}$ increments $\left(\mathrm{GABA}_{\mathrm{A}}\right)$ and between $-5 \mathrm{mV}$ and $15 \mathrm{mV}$ (AMPA-NMDA). When corrected for the liquid junction potential, the average $\mathrm{E}_{\mathrm{rev}}$ for $\mathrm{GABA}_{\mathrm{A}}$ was $-49.2 \pm 1.4 \mathrm{mV}$ and $4.8 \pm 1.5 \mathrm{mV}$ for AMPA-NMDA, close to the predicted values for our internal solution.

Trains of 5 light pulses ( $5 \mathrm{~ms}$ each) at $10 \mathrm{~Hz}$ were used to determine the short-term dynamics of BLA-EPSCs onto PYR, FS-PV, and RSNP-SOM neurons. The $10 \mathrm{~Hz}$ frequency was chosen for the following reasons: (1) BLA neurons reliably fire in response to trains of light pulses at this frequency (see Fig. 1C); (2) this frequency is sufficiently high to evoke dynamics changes in BLA-EPSC amplitudes over the course of the train in GC neurons (see Fig. 4); and (3) this frequency is consistent with BLA firing rates observed in alert animals (Fontanini et al., 2009).

Tonic activation of BLA terminal fields was achieved by using a ramp stimulus in which the intensity of LED stimulation, controlled through the LED driver connected to an analog output of the amplifier, was slowly increased from 0 to $0.3 \mathrm{~mW} / \mathrm{mm}^{2}$ over a $6 \mathrm{~s}$ period. Our choice of stimulation was based on recent results showing that repetitive light pulses of constant intensity decrease the efficiency of light stimulation over time (either for terminal field or neuron stimulation) (Lin et al., 2009). The ramp, on the other hand, progressively recruits presynaptic terminals while maintaining the efficiency of ChR2 stimulation. Thus, ramp stimuli avoid confounds due to progressive loss of efficiency of the stimulation over time, providing a better correlate to sustained or "tonic" activation of BLA afferents. An additional bonus of ramp stimulation of terminal fields is that it allows us to achieve asynchronous activation of axon terminals and engage recurrent circuits.

The quality and stability of the experimental conditions were monitored online by measuring the series resistance $\left(R_{s}\right)$ throughout the recordings. Neurons with $\mathrm{R}_{\mathrm{s}}>20 \mathrm{M} \Omega$ or with $\mathrm{R}_{\mathrm{s}}$ that changed $>10 \%$ during the recording were not included in the analysis.

Immunohistochemistry. Recorded slices were fixed in 4\% PFA for 1 week. After that, they were washed in PBS, permeabilized, and blocked in a solution containing PBS, $1 \%$ Triton $\mathrm{X}$, and $10 \%$ fetal serum for $2 \mathrm{~h}$, then incubated overnight at $4^{\circ} \mathrm{C}$ in a solution containing PBS, $0.1 \%$ Triton X, 3\% fetal serum, and either rat anti-somatostatin (1:100, ThermoFisher) or rabbit anti-parvalbumin (1:1000, Swant). GC slices were then rinsed in $\mathrm{PBS}$ and incubated at $25^{\circ} \mathrm{C}$ for $1.5 \mathrm{~h}$ in a solution containing PBS, $0.1 \%$ Triton X, 3\% fetal serum, and either DyLight 405 conjugated goat anti-rat (1:500, Jackson ImmunoResearch Laboratories) or AlexaFluor-405-conjugated goat anti-rabbit (1:500, Invitrogen). BLA slices were incubated overnight at $4^{\circ} \mathrm{C}$ in a solution containing PBS, $0.1 \%$ Triton X, 3\% fetal serum, rabbit anti-GFP (1:1000, Invitrogen) followed by $20 \mathrm{~min}$ in a solution containing PBS and Hoechst 33342 (1:5000, Invitrogen). All slices were then rinsed in PBS and incubated overnight at $4^{\circ} \mathrm{C}$ in a solution containing PBS, $0.1 \%$ Triton X, and streptavidin-Alexa594 (1:1000, Invitrogen). After a final wash in PBS, slices were mounted with Fluoromount-G and imaged with a confocal microscope (Olympus Fluoview).

Data analysis. Data are presented as mean \pm SE. Normality of data distribution was verified with the Kolmogorov-Smirnov test. Statistical significance was determined with two-tailed, unpaired $t$ tests. Significant differences in cumulative distributions were assessed using two-sample Kolmogorov-Smirnov tests. Input resistance was calculated from the linear portion of the voltage response to a $-50 \mathrm{pA}$ current injection. To test for significant differences in the proportion of BLA-responsive neurons, $\chi^{2}$ for contingency, Pearson correction, was applied. Eventtriggered average of light-evoked BLA-EPSC was used to align BLAEPSC onset and calculate the average amplitude of the light-evoked response. Latencies of BLA-evoked responses were calculated from the onset of the phasic $5 \mathrm{~ms}$ light pulse. The rise time of EPSCs evoked by stimulation of BLA afferents was computed from the onset of the response to the current peak, and decay time constant was calculated by fitting a single exponential function from the current peak as it decayed to baseline. Total synaptic charge was calculated by integrating each current trace.

The short-term plasticity of BLA-EPSCs in response to trains of light pulses was quantified by normalizing the amplitude of each BLA-EPSC in the train to the first BLA-EPSC. This analysis was performed on each recorded neuron. Only after normalizing BLA-EPSC, amplitudes within each recorded neurons normalized data were averaged within each group. Data are presented as normalized amplitude over the number of BLA-EPSC in the train. Statistical comparisons, one-way ANOVA, and post hoc unpaired $t$ test were performed to assess possible differences within group of neurons due to laminar location.

For ramp experiments, synaptic charge was calculated for $6 \mathrm{~s}$ in each epoch (baseline, ramp, and after ramp). Spontaneous events were quantified during the $6 \mathrm{~s}$ period immediately before the ramp (baseline), the $2 \mathrm{~s}$ period before the end of the ramp (ramp), and the $2 \mathrm{~s}$ period after the ramp (after ramp).

Where appropriate, the Bonferroni correction was applied for multiple comparisons. For all statistical tests, $p$ values $\leq 0.05$ were considered significant.

Solutions. ACSF contained the following (in mM): $126 \mathrm{NaCl}, 3 \mathrm{KCl}, 25$ $\mathrm{NaHCO}_{3}, 1 \mathrm{NaHPO}_{4}, 2 \mathrm{MgSO}_{4}, 2 \mathrm{CaCl}_{2}, 14$ dextrose. Internal solution is as follows (in mM): $100 \mathrm{~K}$-Glu, $20 \mathrm{KCl}, 10 \mathrm{~K}$-HEPES, $4 \mathrm{Mg}$-ATP, 0.3 Na-GTP, 10 Na-phosphocreatine, $0.4 \%$ biocytin $\left(\mathrm{V}_{\text {rev }}\left[\mathrm{Cl}^{-1}\right]=-49.8\right.$ $\mathrm{mV})$. The $\mathrm{pH}$ of the internal solution was adjusted to 7.35 with $\mathrm{KOH}$ and the osmolarity was adjusted to $295 \mathrm{mOsm}$ with sucrose. In a set of experiments designed to investigate the excitatory and inhibitory components of BLA-evoked responses with single light pulses (see Fig. 5) or ramps (see Fig. 6), the internal solution contained the following (in mM): 20 $\mathrm{KCl}, 100$ Cs-sulfate, $10 \mathrm{~K}$-HEPES, $4 \mathrm{Mg}$-ATP, $0.3 \mathrm{Na}$-GTP, $10 \mathrm{Na}$ phosphocreatine, 3 QX-314 (Tocris Bioscience), $0.2 \%$ biocytin $\left(\mathrm{V}_{\text {rev }}\right.$ $\left.\left[\mathrm{Cl}^{-1}\right]=-49.3 \mathrm{mV}\right)$. To promote spontaneous activity for ramp experiments, we used a modified ACSF composed of the following (in $\mathrm{mM}$ ): $124 \mathrm{NaCl}, 3.5 \mathrm{KCl}, 0.5 \mathrm{MgCl}_{2}, 1.25 \mathrm{NaHPO}_{4}, 26 \mathrm{NaHCO}_{3}, 1 \mathrm{CaCl}_{2}, 14$ dextrose. For ramp experiments in which the overall activation of $\mathrm{GC}$ was investigated without separating excitatory and inhibitory currents (see Fig. 6), we used an internal solution that mimicked the reversal potential for excitation and inhibition in intact animals containing the following (in mM): $4 \mathrm{KCl}, 116 \mathrm{~K}$-Glu, $10 \mathrm{~K}$-HEPES, $4 \mathrm{Mg}$-ATP, $0.3 \mathrm{Na}$-GTP, 10 Na-phosphocreatine, $0.4 \%$ biocytin $\left(\mathrm{V}_{\text {rev }}\left[\mathrm{Cl}^{-1}\right]=-91.6 \mathrm{mV}\right)$. In experiments in which pharmacological tools were used, all drugs were bath applied at the following concentrations as follows (in $\mu \mathrm{M}$ ): 1 TTX (Tocris Bioscience), 100 4-aminopyridine (4-AP) (Tocris Bioscience), 20 DNQX (Tocris Bioscience), and $50 \mathrm{APV}$ (Tocris Bioscience).

\section{Results}

To determine how BLA afferents activate GC, we relied on a combination of whole-cell, patch-clamp recordings in acute coronal slices of GC and optogenetic tools. BLA neurons were infected with a viral vector carrying a construct in which the light-gated conductance ChR2 was coexpressed with EYFP. The construct was packaged in an adeno-associated virus serotype 9 and injected in the BLA of young rats (for rationale and procedures, see Materials and Methods). With this approach, we were able to express the light-gated conductance in BLA terminal fields in GC and stimulate them selectively using light pulses. The coexpression of EYFP and ChR2 allowed us to visually confirm the location of the injection site (Fig. 1A). To assess sufficient expression of the light-gated protein in BLA neurons, we prepared acute slices containing BLA from a group of injected animals, recorded from BLA neurons (Fig. $1 B$ ), and verified that light stimuli could drive them above threshold for action potential generation (Fig. $1 C)$. We also verified the reliability of action potential generation in BLA neurons in response to trains of brief $(5 \mathrm{~ms}$ at $0.3 \mathrm{~mW} /$ $\mathrm{mm}^{2}$ ) light pulses delivered at different frequencies (Fig. 1C). LED phasic stimulation reliably drove BLA neurons $(n=19)$ above threshold across a range of frequencies from 5 to $20 \mathrm{~Hz}$ 
A

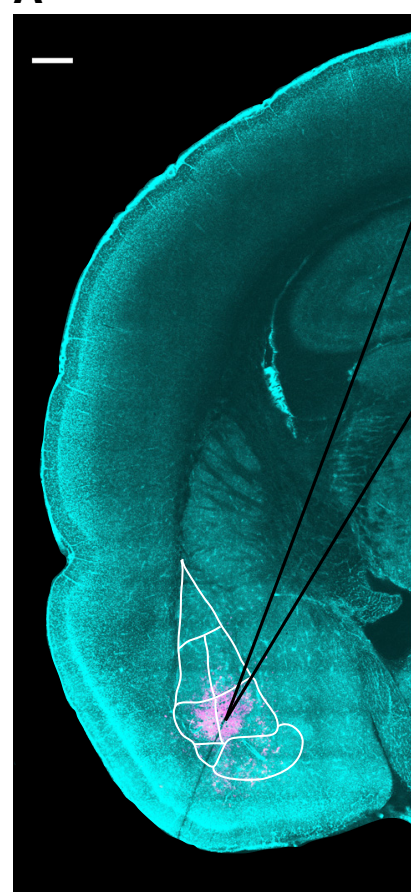

B
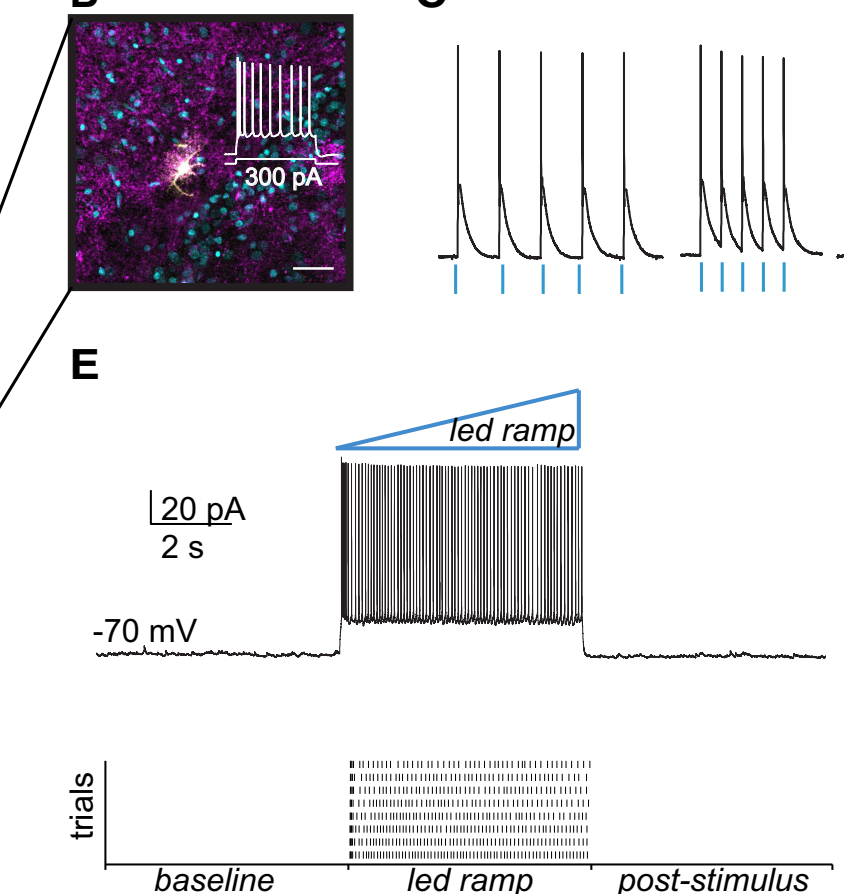

D

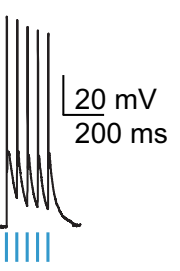

$\mathbf{F}$

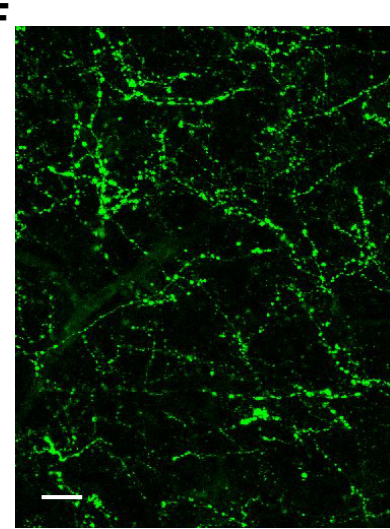

Figure 1. Expression of ChR2-EYFP in BLA. $\boldsymbol{A}$, Representative BLA section showing expression of ChR2-EYFP: Magenta represents ChR2-EYFP. Cyan represents Hoechst. Scale bar, 500 $\mu$ m. $\boldsymbol{B}$, Enlargement of area indicated on left containing recorded neuron: Magenta represents ChR2-EYFP. Cyan represents Hoechst. Yellow represents biocytin. Scale bar, $40 \mu \mathrm{m}$. Inset, Firing pattern of recorded neuron in response to a $300 \mathrm{pA}, 800 \mathrm{~ms}$ depolarizing current step. C, Light-evoked action potentials from neuron shown in $\boldsymbol{B}$. Stimulus intensity $0.3 \mathrm{~mW} / \mathrm{mm}{ }^{2}$. D, Spiking fidelity of BLA neurons in response to light stimuli from 5 to $20 \mathrm{~Hz}$. $\boldsymbol{E}$, Top, Representative BLA neuron firing tonically in response to 6 s ramp of increasing LED power. Bottom, Raster plot of spiking of BLA neuron from top in different trials. Each tic mark represents a spike. $\boldsymbol{F}$, Image of labeled BLA fibers in GC: Green represents ChR2-EYFP. Scale bar, $20 \mu \mathrm{m}$.

(Fig. 1D; spike fidelity percentage: $5 \mathrm{~Hz}, 99.0 \pm 4.4 \%$; $10 \mathrm{~Hz}$, $99.4 \pm 2.0 \% ; 20 \mathrm{~Hz}, 96.9 \pm 11.9 \%)$. To elicit tonic firing in BLA neurons and avoid pulsatile stimulation, we used ramping stimuli, in which the intensity of the LED was slowly increased over a period of $6 \mathrm{~s}$ (Fig. 1E). This mode of stimulation has the ability to drive the light gated channels over long periods of time (Adesnik and Scanziani, 2010) (Fig. 1E). LED ramping stimuli reliably depolarized BLA neurons and led them to fire action potentials throughout the entire $6 \mathrm{~s}$ stimulation period (Fig. $1 E ; n=5$ ) (Faber and Sah, 2002). Finally, we verified that the BLA axons in GC expressed the ChR2-EYFP construct and that no retrograde labeling occurred by imaging and analyzing coronal slices containing GC (Fig. $1 F$; see Fig. $3 A$ ). These data indicate that we obtained sufficient expression of our construct in the BLA to effectively drive neurons above threshold using different patterns of light stimulation. In addition, we show that BLA axons in GC also express the light-gated conductance.

\section{Target-specificity of BLA input}

To study the synaptic organization of the BLA-GC connection, we combined optogenetic stimulation with patch-clamp recordings from visually identified neurons in the aGC. Most studies identified excitatory neurons as the main target of amygdalocortical projections (Paré et al., 1995); therefore, we initially analyzed EPSCs (BLA-EPSCs) in regular spiking neurons with pyramidal morphology (PYR). Biocytin fills and post hoc reconstructions were used to confirm the morphology and location of recorded neurons (Fig. $2 A$ ). Brief ( $5 \mathrm{~ms}$ at $0.3 \mathrm{~mW} / \mathrm{mm}^{2}$ ) phasic pulses of LED stimulation resulted in fast inward currents in PYR neurons (Fig. 2B). The monosynaptic nature of the BLA-EPSC recorded from PYR neurons was verified by sequential and addi- tive bath application of TTX and the A-type potassium channel blocker 4-AP. TTX eliminated evoked currents, consistent with a blockade of the light activation of sodium conductance in BLA terminal fields. The addition of 4-AP, which lengthens the depolarization of presynaptic terminals evoked by light pulses, allowed for a partial recovery of the BLA-EPSC (Fig. $2 B$; PYR, $n=$ $10)$. These experiments indicate that the light-evoked response is not driven by the recurrent circuit but is due to the selective activation of BLA afferents. BLA-EPSCs onto PYR neurons were completely eliminated by bath application of the NMDA and AMPA receptor blockers APV and DNQX, indicating that, in addition to being monosynaptic, BLA-EPSCs are glutamatergic (Fig. 2B; PYR, $n=10$ ) (Cruikshank et al., 2010).

To investigate whether BLA afferents make functional synaptic contacts with other types of neurons in aGC, whole-cell recordings were obtained from inhibitory neurons, which were identified online by their firing pattern in response to steady-state current injections and by their nonpyramidal morphology under DIC optics. Post hoc immunohistochemistry for specific GABAergic neuron markers was used to determine whether the biocytin-filled inhibitory neurons belonged to one or more subpopulations. Recorded neurons belonged to two nonoverlapping populations of inhibitory neurons: fast-spiking, parvalbumin-expressing neurons (FS-PV; Fig. 2C) and regular-spiking, nonpyramidal, somatostatin-expressing neurons (RSNP-SOM; Fig. 2E). LED activation of BLA afferents ( $5 \mathrm{~ms}$ at 0.3 $\mathrm{mW} / \mathrm{mm}^{2}$ ) evoked EPSCs in both populations of inhibitory neurons (Fig. 2D; FS-PV; Fig. 2F; RSNP-SOM). As with PYR, we verified the monosynaptic nature of the BLA-EPSC by perfusing TTX to block spiking, and assessing the recovery of responses evoked by light stimuli delivered in the presence of 4-AP (Fig. $2 D$; FS-PV, $n=8$; Fig. $2 F$; RSNP-SOM, $n=8$ ). These evoked currents also represent direct 
A
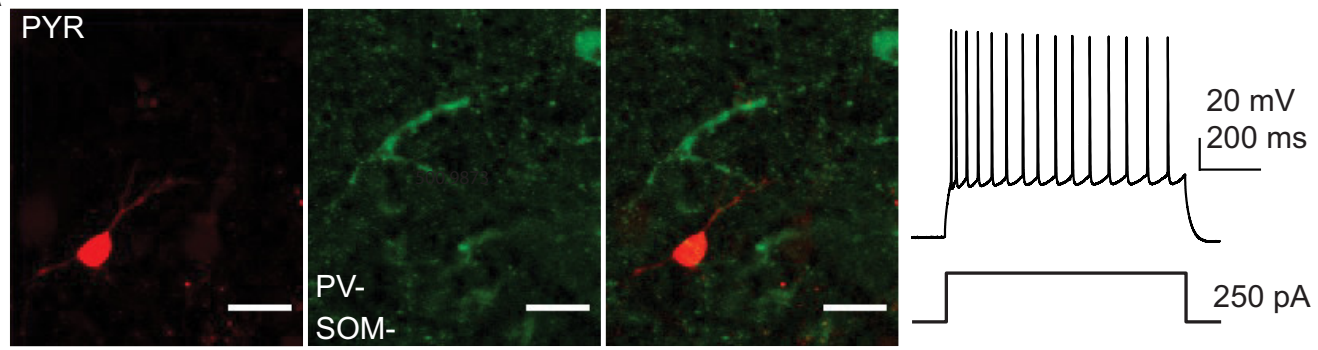

C
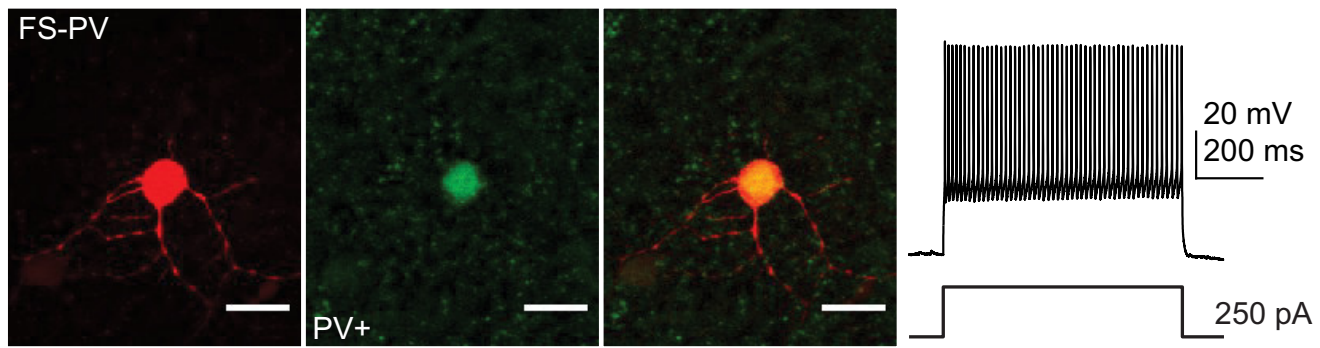

E
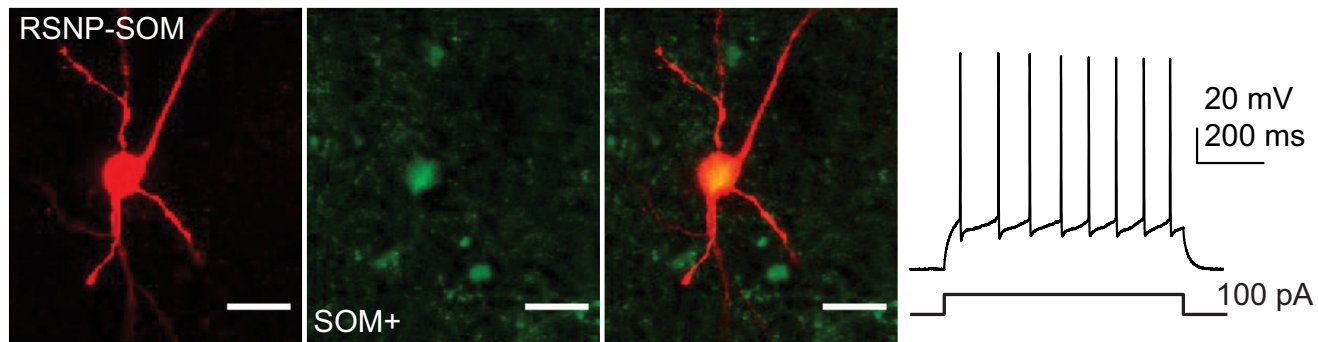

B

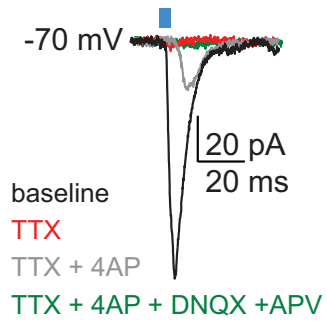

D

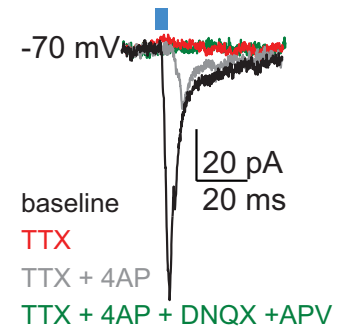

$\mathbf{F}$

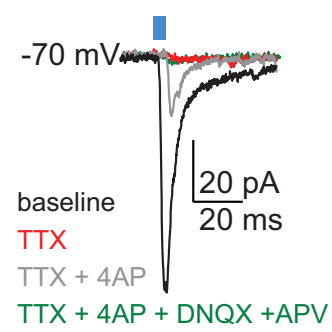

Figure 2. Monosynaptic BLA input onto excitatory and inhibitory neurons in aGC. A, Left, Post hoc reconstruction of recorded PYR neuron (left: biocytin; middle: somatostatin; right: merge). Neurons with PYR morphology were negative for both SOM and PV (data not shown). Scale bar, $20 \mu \mathrm{m}$. Right, Firing pattern of recorded neuron on left in response to a $250 \mathrm{pA}, 800 \mathrm{~ms}$ current step. B, Sample traces of light-evoked BLA-EPSC in PYR: Black represents control trace. Red represents TTX. Gray represents TTX/4AP. Green represents TTX/4AP/DNQX/APV. C, Left, Post hoc reconstruction of recorded FS-PV neuron (left: biocytin; middle: parvalbumin; right: merge). Scale bar, $20 \mu \mathrm{m}$. Right, Firing pattern of recorded neuron on left in response to a $250 \mathrm{pA}, 800 \mathrm{~ms}$ current step. D, Sample traces of light-evoked BLA-EPSC in FS-PV: Black represents control trace. Red represents TTX. Gray represents TTX/4AP. Green represents TTX/4AP/DNQX/APV. E, Left, Post hoc reconstruction of recorded RSNP-SOM neuron (left: biocytin; middle: somatostatin; right: merge). Scale bar, $20 \mu \mathrm{m}$. Right, Firing pattern of recorded neuron on left in response to a $100 \mathrm{pA}, 800 \mathrm{~ms}$ current step. $\boldsymbol{F}$, Sample traces of light-evoked BLA-EPSC in RSNP-SOM: Black represents control trace. Red represents TTX. Gray represents TTX/4AP. Green represents TTX/4AP/DNQX/APV.

glutamatergic input from BLA because they were fully eliminated by bath application of APV and DNQX (Fig. $2 D$; FS-PV, $n=8$; Fig. 2F; RSNP-SOM, $n=8)$. These data demonstrate that BLA can effectively recruit both feedforward excitatory and inhibitory circuits in aGC.

\section{Laminar specificity of BLA input}

Analysis of the fluorescence intensity profile of ChR2-EYFP highlighted a distinct laminar distribution of BLA afferents in aGC. The density of axonal fibers was greater in superficial layers compared with deep layers (Fig. 3A). As this difference may underlie layer-specific connectivity and possibly synaptic properties, we grouped recorded neurons by type and laminar location to ascertain possible differences (Fig. $3 B-D$ ). Because aGC lacks of a visible layer IV, a fundamental landmark for laminar studies in neocortex, we adopted a conservative approach to group our neurons. Neurons were classified as belonging to supragranular (sGC) or infragranular (iGC) layers depending on whether they were superficial or deep relative to an ideal line extending from the fading layer IV in the adjacent dysgranular GC. The latencies of BLA-EPSCs in all neuron types in both layers were consistent with monosynaptic input, indicating that BLA afferents make direct synaptic contact with all three neuron types in both sGC and iGC (Table 1). The proportion of PYR, FS-PV, and RSNP-
SOM neurons responding to photoactivation of BLA afferents was significantly larger in sGC than iGC (Fig. 3E-G; Table 1; percentage response; $\chi^{2}$ for contingency: PYR, $p<10^{-4}$; FS-PV, $p<10^{-3}$; RSNP-SOM, $\left.p<0.02\right)$. This difference in connectivity is consistent with the density of BLA afferents in the superficial and deep layers of aGC. The amplitude of BLA-EPSCs onto PYR neurons was greater in sGC, but BLA-EPSCs onto FS-PV or RSNP-SOM neurons did not differ significantly between superficial and deep layers (Fig. 3E-G; Table 1; amplitude: PYR, $p<$ 0.002 ; FS-PV, $p=0.9$; RSNP-SOM, $p=0.4$ ). Similarly, the charge of BLA-EPSCs was larger for PYR neurons in sGC than iGC, whereas no differences were observed for BLA-EPSCs onto FS-PV or RSNP-SOM neurons across layers (Fig. 3E-G; Table 1; charge: PYR, $p<10^{-4}$; FS-PV, $p=0.9$; RSNP-SOM, $p=0.3$ ).

These results indicate that, although BLA afferents synapse onto excitatory and inhibitory neurons in both the superficial and deep layers of aGC, this input shows laminar-specific connectivity, and in the case of PYR neurons layer-specific synaptic strength.

\section{Short-term dynamics of BLA input}

Activating BLA terminal fields in aGC with a single LED pulse demonstrated that this input has laminar and target-specific properties. We next investigated whether repetitive stimulation 


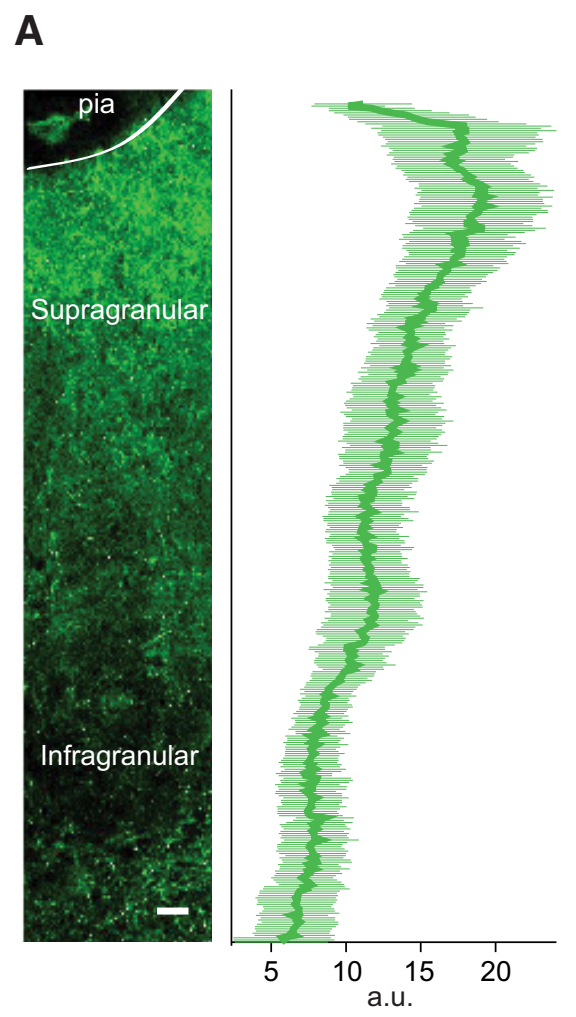

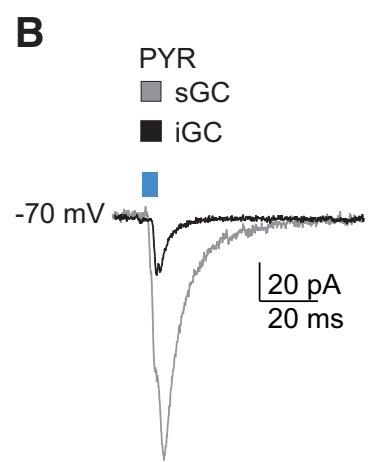

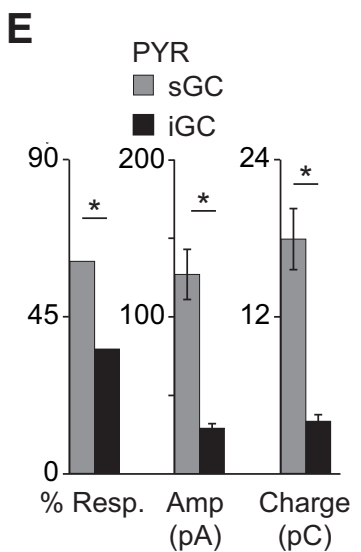

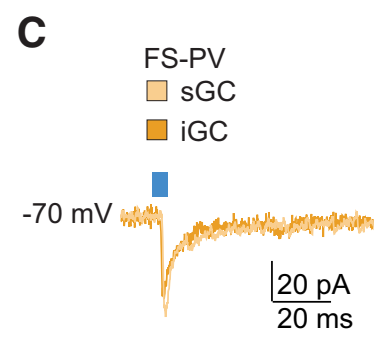

$\begin{aligned} & \text { RSNP-SOM } \\ & \square \text { sGC } \\ & \square \text { iGC }\end{aligned}$

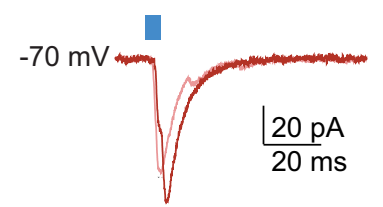

F

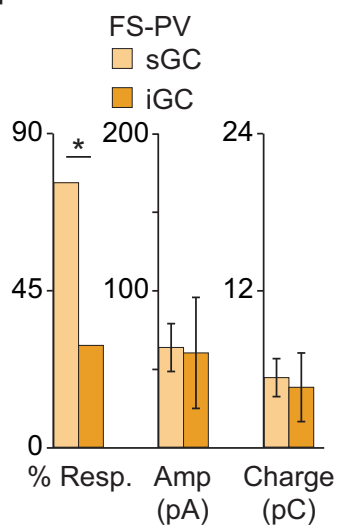

G

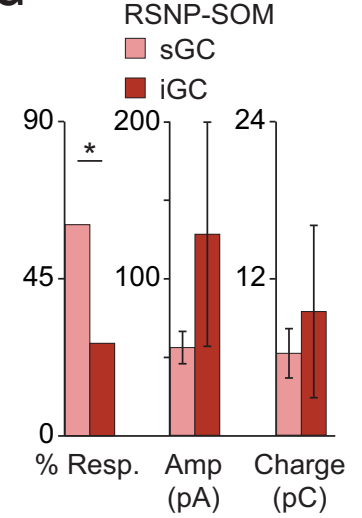

Figure 3. Laminar differences in BLA input to sGC and iGC. $A$, Left, Expression of ChR2-EYFP in BLA terminal fields in aGC. Right, Calibration curve of GFP intensity across lamina (see Materials and Methods). Scale bar, $50 \mu \mathrm{m}$. Dark green line indicates average intensity. Shaded borders represent SD. B, Sample traces of light-evoked BLA-EPSCs in PYR neurons in sGC (gray) and iGC (black). C, Sample traces of light-evoked BLA-EPSCs in FS-PV neurons in SGC (light yellow) and iGC (dark yellow). D. Sample traces of light-evoked BLA-EPSCs in RSNP-SOM neurons in sGC (pink) and iGC (red). $\boldsymbol{E}$, Response percentage, average amplitude, and average charge of PYR neurons in sGC (gray) and iGC (black). $\boldsymbol{F}$, Response percentage, average amplitude, and average charge of FS-PV neurons in sGC (light yellow) and iGC (dark yellow). G, Response percentage, average amplitude, and average charge of RSNP-SOM neurons in sGC (pink) and iGC (red). Data are mean \pm SE. *Significant difference.

Table 1. BLA-EPSC response properties ${ }^{a}$

\begin{tabular}{|c|c|c|c|c|c|c|}
\hline & \multicolumn{3}{|l|}{ sGC } & \multicolumn{3}{|l|}{ iGC } \\
\hline & PYR & FS-PV & RSNP-SOM & PYR & FS-PV & RSNP-SOM \\
\hline Response percentage & 81 of $132,61.4 \%$ & 28 of $37,75.7 \%$ & 21 of $36,58.3 \%$ & 33 of $93,35.5 \%$ & 7 of $23,30.4 \%$ & 9 of $30,30.0 \%$ \\
\hline Amplitude (pA) & $122 \pm 14.8, n=82$ & $63.4 \pm 15.2, n=28$ & $48.2 \pm 8.6, n=21$ & $46.3 \pm 17.7, n=32$ & $58.1 \pm 35.7, n=6$ & $85.2 \pm 43.8, n=9$ \\
\hline Charge $(p C)$ & $1.7 \pm 0.2, n=81$ & $0.5 \pm 0.1, n=26$ & $0.5 \pm 0.1, n=20$ & $0.4 \pm 0.05, n=29$ & $0.6 \pm 0.3, n=4$ & $1.1 \pm 0.6, n=7$ \\
\hline Latency (ms) & $2.6 \pm 0.06, n=82$ & $2.7 \pm 0.1, n=28$ & $2.4 \pm 0.1, n=21$ & $2.9 \pm 0.1, n=32$ & $2.8 \pm 0.3, n=6$ & $2.7 \pm 0.2, n=9$ \\
\hline PPR (EPSC2/EPSC1) & $0.86 \pm 0.03, n=28$ & $0.95 \pm 0.09, n=15$ & $0.99 \pm 0.1, n=10$ & $0.98 \pm 0.08, n=16$ & $0.52 \pm 0.1, n=2$ & $0.83 \pm 0.06, n=6$ \\
\hline SSR (EPSC5/EPSC1) & $0.73 \pm 0.03, n=27$ & $0.88 \pm 0.07, n=15$ & $0.85 \pm 0.1, n=9$ & $0.69 \pm 0.04, n=14$ & $0.67 \pm 0.4, n=2$ & $0.73 \pm 0.07, n=6$ \\
\hline
\end{tabular}

${ }^{a}$ Average response percentage, amplitude, charge, PPR, and steady-state ratio of BLA-EPSCs onto PYR, FS-PV, and RSNP-SOM neurons in sGC and iGC. Data are mean \pm SE.

of BLA afferents would reveal differences in the short-term dynamics of BLA input across aGC laminae. To do this, we delivered a train of 5 brief light pulses at $10 \mathrm{~Hz}$ and recorded BLA-EPSCs in PYR, FS-PV, and RSNP-SOM neurons in sGC and iGC (Fig. $4 A-C)$. We chose $10 \mathrm{~Hz}$ because in our experimental conditions BLA neurons reliably fire action potentials in response to train of light pulses at this frequency (Fig. $1 C, D$ ); in addition, it is a frequency consistent with those observed from recordings of BLA neurons in alert rats (Fontanini et al., 2009). Phasic, $10 \mathrm{~Hz}$ activation of $\mathrm{BLA}$ afferents resulted in a paired-pulse ratio (PPR; EPSC2/EPSC1), as well as a steady-state ratio (SSR; EPSC5/ EPSC1) $<1$ in all groups, indicating that BLA synapses onto PYR, FS-PV, and RSNP-SOM cells in both SGC and iGC show shortterm depression in response to repetitive BLA stimulation (Table 1). We then assessed possible laminar differences in BLA-evoked short-term dynamics within each group of neurons. There were no laminar-specific differences in PPR and SSR within each group of neurons (Fig. 4D-F; Table 1; sGC vs iGC: PYR, PPR: $p=$ 0.2 ; SSR: $p=0.4$; FS-PV, PPR: $p=0.1$; SSR: $p=0.7$; RSNP-SOM, PPR: $p=0.4$; SSR: $p=0.3$ ).

Together, these results show that the short-term plasticity of BLA-EPSCs does not differ significantly by neuron group or laminar location.

\section{BLA evokes feedforward excitation and inhibition in GC pyramidal neurons}

PYR neurons are the primary output of cortical circuits to other layers as well as other areas (Allen et al., 1991; Adachi et al., 2013). Our data show that BLA inputs onto them show layer-specific differences in connectivity and synaptic strength. We therefore asked whether BLA stimulation may recruit feedforward excitation and inhibition differently onto PYR neurons located in the superficial and deep layers of aGC. To dissect the contribution of feedforward excitation and inhibition to BLA-evoked responses, 
A

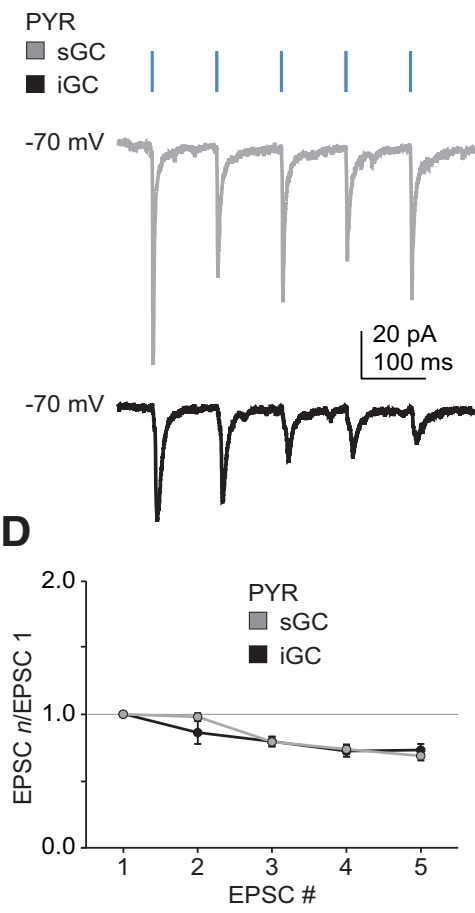

B

\section{FS-PV \\ $\square$ sGC}

$\square$ iGC

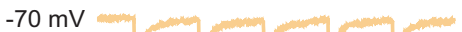
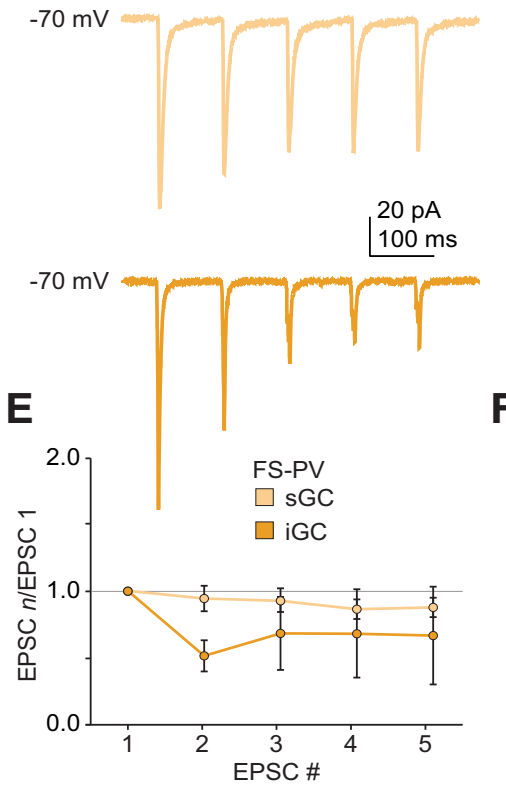

C
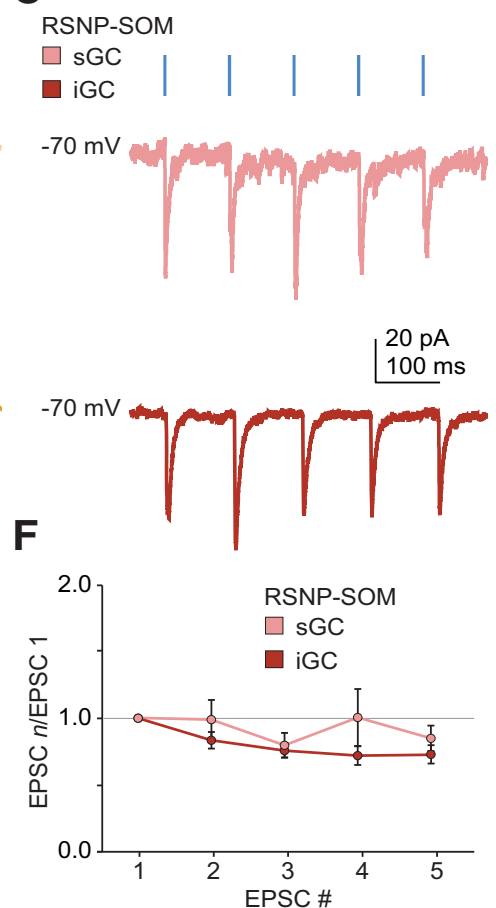

Figure 4. Short-term dynamics of BLA-EPSCs. A, Top, Sample trace of a $10 \mathrm{~Hz}$ train of BLA-EPSCs in sGC PYR neuron (light gray). Bottom, Sample trace of a $10 \mathrm{~Hz}$ train of BLA-EPSCs in iGC PYR neuron (black). B, Top, Sample trace of a $10 \mathrm{~Hz}$ train of BLA-EPSCs in sGC FS-PV neuron (light yellow). Bottom, Sample trace of a $10 \mathrm{~Hz}$ train of BLA-EPSCs in iGC FS-PV neuron (dark yellow). C, Top, Sample trace of a $10 \mathrm{~Hz}$ train of BLA-EPSCs in sGC RSNP-SOM neuron (pink). Bottom, Sample trace of a $10 \mathrm{~Hz}$ train of BLA-EPSCS in iGC RSNP-SOM neuron (red). D, Average ratio of EPSCn/EPSC1 for $10 \mathrm{~Hz}$ BLA stimulation in PYR neurons in SGC (gray) and iGC (black). E, Average ratio of EPSC $/$ /EPSC1 for $10 \mathrm{~Hz}$ BLA stimulation in FS-PV neurons in sGC (light yellow) and iGC (dark yellow). $\boldsymbol{F}$, Average ratio of EPSCn/EPSC1 for $10 \mathrm{~Hz}$ BLA stimulation in RSNP-SOM neurons in SGC (pink) and iGC (red). Data are mean \pm SE.

we delivered single light pulses to activate BLA terminal fields while holding recorded PYR neurons at the reversal potentials for inhibitory or excitatory conductance (Fig. 5A). This approach allowed us to isolate monosynaptic excitatory, and disynaptic inhibitory evoked responses in each recorded neuron. Single pulse activation of BLA afferents resulted in a short latency EPSC (monosynaptic) and a longer-latency IPSC (disynaptic; Table 2; Fig. $5 A, B$; latency: EPSC vs IPSC, sGC: $p<10^{-4}$, iGC: $p<$ $\left.10^{-4}\right)$. The amplitude of both BLA-EPSCs and BLA-IPSCs onto PYR neurons was largest in sGC (EPSC; Fig. 5C; Table 2; amplitude: $s$ GC vsiGC, $p<0.01$; IPSC; Fig. 5D; Table 2; amplitude: $s G C$ vs iGC, $p<0.01)$. The differences in EPSC and IPSC amplitude were consistent with differences in the charge of the events (EPSC; Fig. $5 C$; Table 2; charge: sGC vs iGC, $p<0.03$; IPSC; Fig. $5 D$; Table 2; charge: sGC vs iGC, $p<0.04$ ). The ratio of BLAevoked excitatory and inhibitory charge flowing through PYR neuron membranes was close to 1 and did not differ significantly between layers (Fig. $5 E$; E/I ratio: $\mathrm{sGC}, 0.95 \pm 0.23, n=20$; iGC, $0.90 \pm 0.2, n=10 ; p=0.9)$.

These results indicate that BLA terminal field activation with single stimuli evokes larger EPSC and IPSC in sGC compared with iGC. However, BLA activation recruits balanced feedforward excitation and inhibition in PYR neurons located in both sGC and iGC.

\section{Tonic activation of BLA afferents in GC}

Phasic pulses may recruit the aGC circuitry different from tonic and continuous activation of BLA afferents. In the next set of experiments, we investigated how tonic BLA activity modulates spontaneous synaptic activity onto neurons in the superficial and deep layers of aGC. To mimic continuous fir- ing, we used a 6-s-long ramping light stimulus (see Materials and Methods). We recorded BLA-evoked spontaneous postsynaptic currents (sPSCs) from PYR, FS-PV, and RSNP-SOM neurons in sGC (Fig. $6 A, D, G$ ) and iGC (Fig. 6B,E,H) before, during, and after the ramping stimulus to examine how sustained BLA activity affects circuit activity. In this set of experiments, we used an internal solution that mimicked the chloride reversal potential observed in intact animals $(-91 \mathrm{mV}$; see Materials and Methods) and maintained neurons in voltage clamp at $-70 \mathrm{mV}$, close to their resting membrane potential. This approach was used to test whether tonic BLA activity could modulate spontaneous synaptic currents on GC neurons in conditions as close to physiological as can be achieved in a slice preparation. There were significant increases in amplitude and instantaneous frequency of sPSCs onto PYR neurons in both layers of aGC during the ramp (sGC; Fig. $6 A, B$; Table $3, n=13$, amplitude: baseline vs ramp, $p<10^{-4}$; instantaneous frequency: baseline vs ramp, $p<10^{-4}$; iGC; Fig. 6C,D; Table 3; $n=9$, amplitude: baseline vs ramp, $p<$ 0.01 ; instantaneous frequency: baseline vs ramp, $\left.p<10^{-4}\right)$. The increase in total synaptic charge due to the ramp was significantly greater in PYR neurons recorded in sGC than in iGC (Table 3; Fig. $6 M$; percentage change from baseline, $p<0.04$ ). Thus, tonic BLA activity drives the circuit in sGC more intensely than in iGC. This effect is consistent with the laminar differences in connection probability observed in aGC (Fig. 3).

We also looked at how tonic activation of BLA afferents affects spontaneous synaptic activity in FS-PV and RSNP-SOM neurons in sGC and iGC. In sGC, ramp activation of BLA terminals resulted in a significant increase of sPSC instantaneous frequency, but not amplitude onto FS-PV neurons, and a significant increase of both instantaneous frequency and amplitude of sPSCs onto 
RSNP-SOM neurons (sGC; FS-PV: Table 3; Fig. 6E, F; amplitude: baseline vs ramp, $p=0.5$; instantaneous frequency: baseline vs ramp, $p<10^{-4}$; RSNP-SOM: Table 3; Fig. 6I,J; amplitude: baseline vs ramp, $p<$ $10^{-4}$; instantaneous frequency: baseline vs ramp, $p<10^{-4}$ ). In iGC, ramp activation of BLA terminals resulted in a significant increase of both sPSC amplitude and instantaneous frequency onto FS-PV neurons, and a significant increase of sPSC instantaneous frequency, but not sPSC amplitude onto RSNP-SOM neurons (iGC; FS-PV: Table 3; Fig. 6G, H; amplitude: baseline vs ramp, $p=10^{-2}$; instantaneous frequency: baseline vs ramp, $p<10^{-4}$; RSNP-SOM: Table 3; Fig. $6 K, L$; amplitude: baseline vs ramp, $p=0.7$; instantaneous frequency: baseline vs ramp, $p<10^{-4}$ ). Importantly, unlike for PYR neurons, the change in total charge evoked by the ramp onto FS-PV and RSNP-SOM did not show laminar differences (Fig. 6M; Table 3; percentage Change from baseline, sGC vs iGC: FSPV,$p=0.6$; RSNP-SOM: $s$ GC vs iGC, $p=$ $0.5)$. Together, these results suggest that tonic activation of BLA afferents modulates spontaneous activity in a layerspecific fashion only in PYR neurons.

Tonic BLA activity engages feedforward excitatory and inhibitory circuits

The data presented so far highlight that BLA inputs onto PYR neurons show laminar specificity for connectivity, amplitude, and modulation of spontaneous activity by tonic BLA activity. Differently, BLA inputs onto FS-PV and RSNP-SOM show laminar differences in connectivity, but not for the other parameters we analyzed. We therefore focused on PYR neurons and asked whether their location in sGC or iGC affects how ramps modulate the spontaneous excitatory and inhibitory synaptic input onto them.

To isolate excitatory and inhibitory currents from the same recorded PYR neurons, we used the same internal solution as for the experiments shown in Figure 5 (Fig. 7A: sGC; Fig. 7C: iGC). The cumulative distributions of amplitude and frequency for spontaneous inhibitory (sIPSC) and excitatory (sEPSC) currents before and during the ramping stimulus were compared (Fig. $7 B$ : sGC; Fig. 7D: iGC). PYR neurons in sGC showed a significant increase in amplitude and instantaneous frequency of sIPSCs during sustained activation of BLA afferents (Fig. 7B; Table 4; sGC, $n=14$, sIPSC: amplitude: baseline vs ramp, $p<10^{-3}$; instantaneous frequency: baseline vs ramp, $p<10^{-3}$ ). In addition, amplitude and instantaneous frequency of sEPSCs were significantly elevated during the ramp (Fig. $7 B$; Table 4; sGC, $n=13$, sEPSC: amplitude: baseline vs ramp, $p<10^{-3}$; instantaneous frequency: baseline vs ramp; $p<10^{-3}$ ). The increases in amplitude and instantaneous frequency of sIPSCs and sEPSCs in sGC during ramp activation of BLA afferents corresponded with a significant increase in both ramp-evoked excitatory and inhibitory charge (Fig. 7E; Table 4; sGC, $n=13$, excitatory [EXC]: baseline vs ramp, $p<0.01$; inhibitory [INH] charge: baseline vs ramp, $p<0.01)$. The ratio of excitatory and inhibitory charge was $<1$ before and during the ramping stimulus (Fig. $7 F$; sGC, $n=$ 13, E/I ratio: baseline, $0.75 \pm 0.06$; ramp, $0.86 \pm 0.12 ; p=0.4$ ). Thus, tonic activation of BLA afferents recruits excitatory and inhibitory circuits in sGC. BLA ramping stimuli had a different effect on aGC circuits from what we observed for single stimuli. Instead of evoking balanced excitatory and inhibitory charge like single BLA stimuli, tonic activation of BLA afferents led to an overall increase in sGC synaptic activity but maintained the bias toward inhibition that was observed before the delivery of the ramping stimulus.

In iGC, ramp activation of BLA afferents increased both amplitude and frequency of sIPSCs, although it only increased the frequency of sEPSCs (Fig. 7D; Table 4; iGC, sIPSC, $n=8$, amplitude: baseline vs ramp, $p<0.009$; instantaneous frequency: baseline vs ramp, $p<10^{-3}$; sEPSC, $n=9$, amplitude: baseline vs ramp, $p=0.2$; instantaneous frequency: baseline vs ramp, $p<$ $\left.10^{-3}\right)$. Similar to sGC, the increases in sIPSC amplitude and frequency resulted in a significant increase in the total inhibitory charge onto iGC PYRs. In addition, the increase in sEPSC instantaneous frequency alone was sufficient to significantly increase the excitatory charge onto iGC PYRs (charge; Fig. 7E; Table 4; iGC, $n=7$, EXC: baseline vs ramp, $p<0.03$; INH: baseline vs 
Table 2. BLA-evoked feedforward excitation and inhibition ${ }^{a}$

\begin{tabular}{|c|c|c|c|c|c|c|}
\hline & sGC-PYR & & & iGC-PYR & & \\
\hline & Latency (ms) & Amplitude (pA) & Charge $(p C)$ & Latency (ms) & Amplitude (pA) & Charge $(p C)$ \\
\hline BLA-EPSC & $14.2 .6 \pm 0.1, n=20$ & $237 \pm 59.6, n=20$ & $4.0 \pm 1.1, n=20$ & $2.8 \pm 0.1, n=10$ & $54.5 \pm 21.9, n=10$ & $2.0 \pm 0.9, n=10$ \\
\hline BLA-IPSC & $6.7 \pm 0.3, n=18$ & $197 \pm 46.8, n=20$ & $5.6 \pm 1.4, n=20$ & $6.4 \pm 0.6, n=10$ & $58.9 \pm 17.9, n=10$ & $2.0 \pm 0.9, n=10$ \\
\hline
\end{tabular}

${ }^{a}$ Average latency amplitude, and charge, of BLA-EPSCs and BLA-IPSCs onto PYR neurons in $S G C$ and $i G C$. Data are mean \pm SE.

ramp, $p<0.04)$. As in sGC, ramp activation of BLA afferents in iGC did not alter the ratio between excitatory and inhibitory charge (Fig. 7F; iGC, $n=7$, E/I ratio: baseline, $0.58 \pm 0.09$; ramp, $0.76 \pm 0.22 ; p=0.3)$, which remained $<1$ before and during the ramping stimulus, suggesting that the circuit in iGC is dominated by inhibition and remains so during sustained activation of BLA afferents. Thus, tonic activation of BLA inputs recruits both excitatory and inhibitory inputs onto PYR neurons in all layers of aGC, but maintaining the bias toward inhibition of aGC spontaneous activity.

When changes in excitatory and inhibitory charge were compared across layers, we found that tonic activation of BLA afferents increased excitation and inhibition onto sGC-PYR neurons significantly more than onto iGC-PYR neurons (Fig. 7G; Table 4; percentage change from baseline, EXC: sGC vs iGC, $p<0.04$; INH: sGC vs iGC, $p<0.02)$. These results are likely explained by the larger connectivity of BLA afferents onto neurons in sGC compared with iGC. Despite larger changes in BLA ramp-evoked excitation and inhibition in sGC, the E/I ratio did not differ significantly between layers, indicating that, despite the differences in connectivity, inhibition remains dominant in both sGC and iGC during tonic activation of BLA afferents (Fig. 7F; E/I ratio: sGC vs iGC, $p=0.7)$.

\section{Tonic activation of BLA afferents engages recurrent circuits in aGC}

Ramp stimulation of terminal fields, in addition to evoking tonic BLA neuron firing, was also shown to elicit asynchronous activation of cortical circuits as it can progressively engage intracortical recurrent connectivity with increasing light intensity (Adesnik and Scanziani, 2010). If recurrent circuits are recruited by the ramp, one would expect that the changes in spontaneous synaptic events observed in PYR neurons would outlast the duration of the ramping stimulus. When examining the effect of tonic BLA activation on spontaneous activity onto PYR neurons after the end of the ramp, we observed that, in both sGC and iGC, the increase in synaptic charge remained significantly elevated relative to baseline (charge: sGC-PYR; Fig. $6 A$; baseline vs after ramp, $p<0.01$; iGC-PYR; Fig. $6 B$; baseline vs after ramp, $p<0.01$ ). Overall, charge was affected more in sGC compared with iGC (Fig. 6C; Table 3; PYR: percentage change from baseline, sGC vs iGC, $p<$ $0.04)$. The maintenance of significant increases in spontaneous synaptic charge after the end of the ramp supports the interpretation that, in addition to directly activating inputs onto PYR neurons, this regimen of BLA activity can drive recurrent circuits in $\mathrm{aGC}$.

We then asked whether the elevated activity after the ramping stimulus relied on changes in excitation, inhibition, or both. In sGC, sEPSC amplitude and frequency and sIPSC frequency remained elevated, whereas sIPSC amplitude returned to baseline (Fig. 7B; Table 4; sGC, sIPSC, $n=14$, amplitude: baseline vs after ramp, $p=0.8$; instantaneous frequency: baseline vs after ramp, $p<10^{-4}$; sEPSC, $n=13$, amplitude: baseline vs after ramp, $p<$ 0.04; instantaneous frequency: baseline vs after ramp, $p<10^{-4}$ ). This resulted in a persistent increase in excitatory charge only
(Fig. 7E; Table 4; charge: sGC, $n=13$, EXC: baseline vs after ramp, $p<0.03$; INH: baseline vs after ramp, $p=0.1$ ).

In iGC, sEPSC frequency and sIPSC amplitude remained elevated after the end of the ramp (Fig. $7 D$; Table 4; iGC, sIPSC, $n=$ 8, amplitude: baseline vs after ramp, $p<0.01$; instantaneous frequency: baseline vs after ramp, $p=0.9$; sEPSC, $n=9$, amplitude: baseline vs after ramp, $p=0.6$; instantaneous frequency: baseline vs after ramp, $\left.p<10^{-4}\right)$. Similar to $\mathrm{sGC}$, only the excitatory charge remained significantly elevated after the ramp (Fig. $7 E$; Table 4; charge: iGC, $n=7$, EXC: baseline vs after ramp, $p<$ 0.01 ; INH: baseline vs after ramp, $p=0.08$ ).

The post-ramp increase in excitatory charge in sGC was significantly greater than in iGC (Fig. $7 E$; Table 4; charge, sGC vs iGC, EXC: $p=0.1$; INH: $p=0.7$; percentage change from baseline; Fig. 7G; Table 4; EXC: sGC vs iGC, $p<0.04$; INH: sGC vs $\mathrm{iGC}, p=0.2)$. Together, these data suggest that tonic activation of BLA afferents can engage recurrent circuits in both the superficial and deep layers of aGC. However, the sGC excitatory circuit is more strongly activated than the one in iGC.

\section{Layer-specific recruitment of recurrent circuits by tonic activation of BLA afferents in aGC}

If ramp stimuli can recruit and excite recurrent circuits, it is possible that this pattern of BLA activity may modulate spontaneous activity of aGC neurons that do not receive direct inputs from BLA. To examine this possibility, we recorded PYR neurons that did not show BLA-evoked EPSCs in response to single light pulses, and quantified the excitatory and inhibitory charge onto them before, during, and after BLA ramp stimulation.

In $\mathrm{sGC}$, only 1 of 6 PYR neurons that did not receive monosynaptic BLA input showed any change in synaptic activity during or after the ramp. Thus, in sGC, PYR neurons that do not receive direct BLA input did not show significant changes in the average excitatory and inhibitory charge either during or following the ramp (Fig. $8 E$; sGC, $n=6$, charge, normalized to baseline: EXC, ramp: $1.08 \pm 0.06$; after ramp: $1.11 \pm 0.07$; baseline vs ramp, $p=0.2$; baseline vs after ramp, $p=0.2$; INH, ramp: $1.06 \pm$ 0.08; after ramp: $1.19 \pm 0.18$; baseline vs ramp, $p=0.4$; baseline vs after ramp, $p=0.3$ ). Together with the results about the BLAsGC connection probability (Fig. 3) and the data shown in Figure 7 , these data suggest that, in $\mathrm{sGC}$, asynchronous recruitment of BLA afferents with ramping stimuli modulates the activity of circuits formed by a large percentage of excitatory and inhibitory neurons driven by monosynaptic BLA inputs (Fig. 3E; Table 1).

Differently, in iGC, $50 \%$ of PYR neurons that did not receive direct BLA input (Fig. 8C) showed a significant increase in both excitatory and inhibitory charge during the ramp. This increase persisted following the ramp (Fig. $8 E$; iGC, $n=10$, charge, normalized to baseline: EXC, ramp: $1.23 \pm 0.06$; after ramp: $1.22 \pm$ 0.08 ; baseline vs ramp, $p<0.01$; baseline vs after ramp, $p<0.04$; INH, ramp: $1.21 \pm 0.06$; after ramp: $1.44 \pm 0.1$; baseline vs ramp, $p<0.01$; baseline vs after ramp, $p<0.01)$. These data indicate that, in the deep layers of aGC, BLA ramp stimuli engage recurrent circuits activated by a small percentage of neurons that re- 

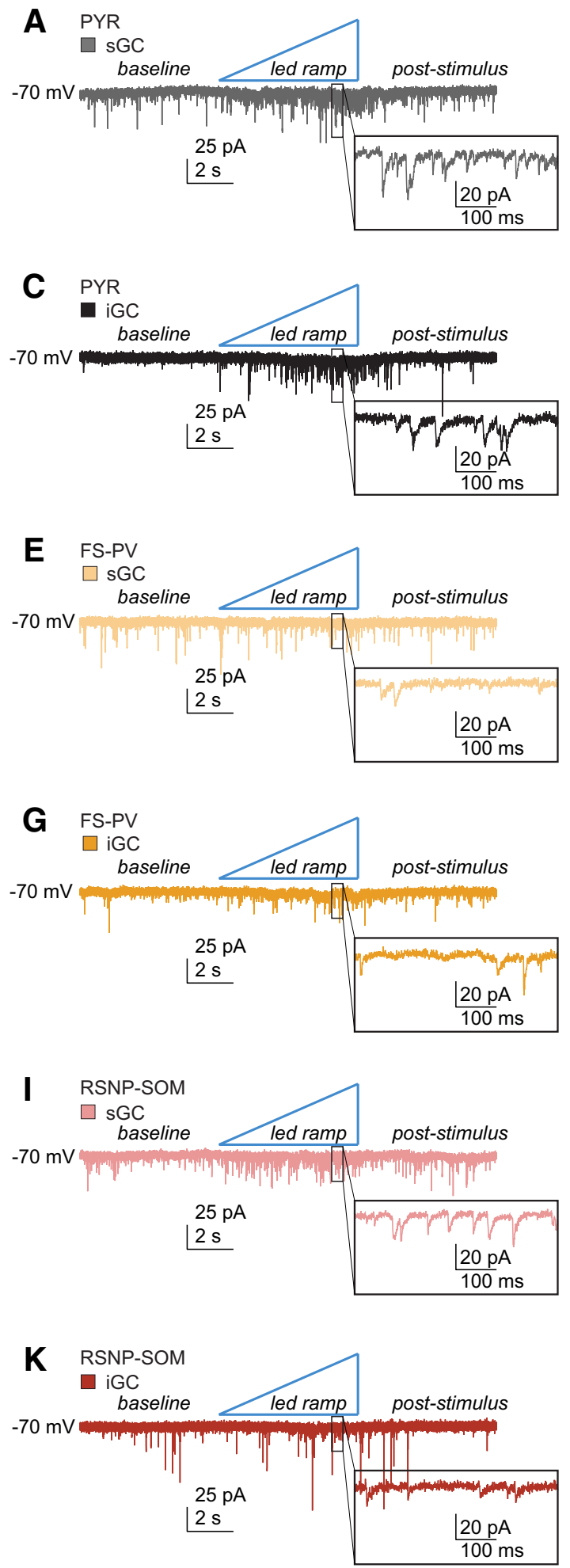

M

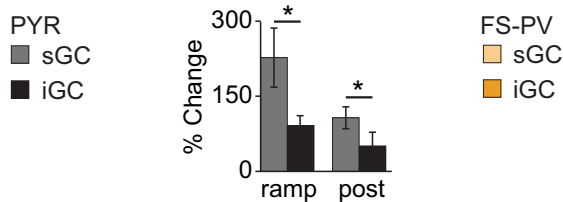

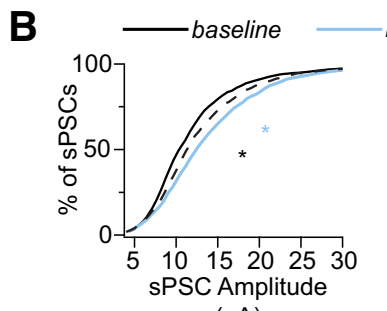

(pA)

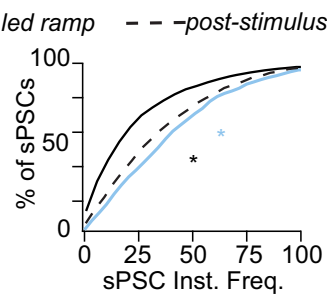

$(\mathrm{Hz})$

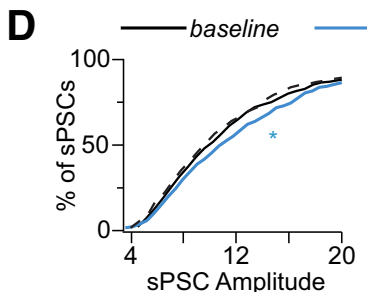

(pA)

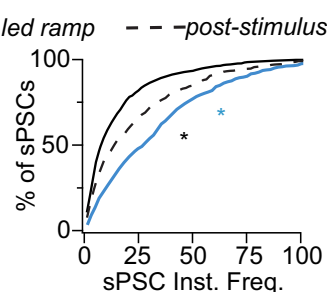

$(\mathrm{Hz})$

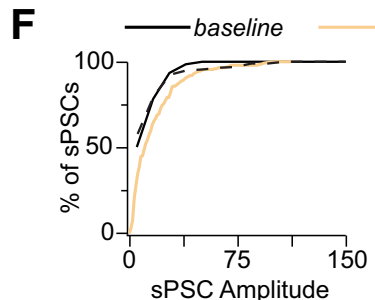

(pA)

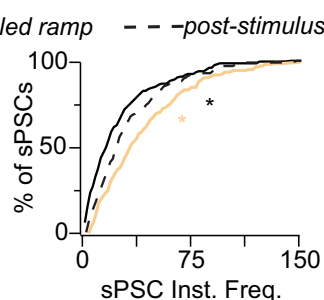

$(\mathrm{Hz})$

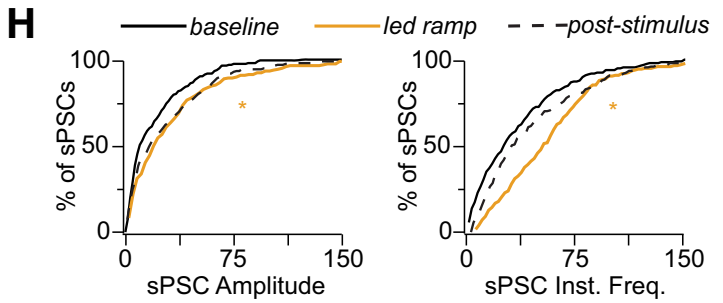

(pA)

$(\mathrm{Hz})$

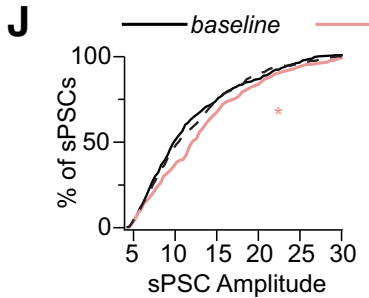

(pA)

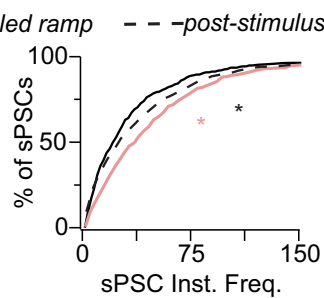

$(\mathrm{Hz})$

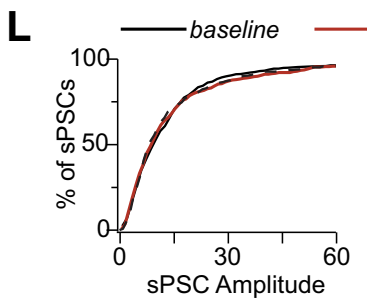

(pA)

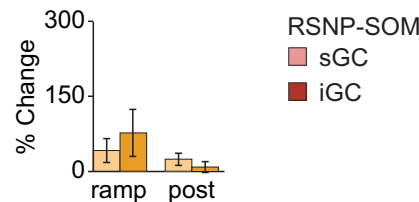

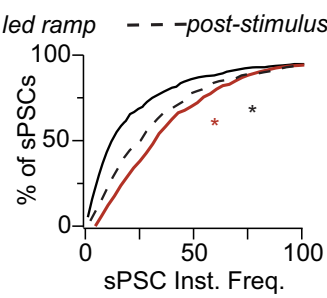

$(\mathrm{Hz})$

Figure 6. Tonic activation of BLA afferents promotes recurrent circuit activity in sGC and iGC. $\boldsymbol{A}$, Top, Sample trace of spontaneous currents recorded from a PYR neuron in sGC (gray). Inset, Stretched portion of the trace included in the vertical rectangle. $B$, CDFs of sPSC amplitude (left) and sEPSC instantaneous frequency (right) in sGCPYR. Black represents baseline. Light blue represents ramp. Dashed line indicates after ramp. C, Sample trace of spontaneous currents recorded from a PYR neuron in iGC (black). Inset, Stretched portion of the trace (Figure legend continues.) 
Table 3. BLA ramp-evoked activity in SGC and iGC ${ }^{a}$

\begin{tabular}{|c|c|c|c|c|c|c|}
\hline & \multicolumn{3}{|l|}{$\mathrm{sGC}$} & \multicolumn{3}{|l|}{$\mathrm{iGC}$} \\
\hline & Baseline & Ramp & After ramp & Baseline & Ramp & After ramp \\
\hline \multicolumn{7}{|l|}{ PYR } \\
\hline $\begin{array}{l}\text { Amplitude (pA) } \\
\mathrm{sGC}(n=15)\end{array}$ & $11.8 \pm 0.2$ & $13.9 \pm 0.2$ & $12.7 \pm 0.2$ & $12.8 \pm 0.30$ & $13.3 \pm 0.3$ & $11.8 \pm 0.3$ \\
\hline $\begin{array}{l}\text { Instantaneous frequency }(\mathrm{Hz}) \\
\mathrm{iGC}(n=10)\end{array}$ & $27.5 \pm 0.8$ & $42.0 \pm 0.9$ & $37.3 \pm 0.9$ & $15.7 \pm 0.6$ & $33.8 \pm 1.0$ & $24.1 \pm 0.9$ \\
\hline $\begin{array}{l}\text { \% change (total charge) } \\
\text { FS-PV }\end{array}$ & - & $209.8 \pm 54.6$ & $98.7 \pm 20.3$ & - & $84.2 \pm 16.8$ & $47.0 \pm 10.1$ \\
\hline $\begin{array}{l}\text { Amplitude (pA) } \\
\operatorname{sGC}(n=2)\end{array}$ & $23.7 \pm 5.5$ & $22.1 \pm 1.3$ & $25.3 \pm 5.5$ & $15.3 \pm 0.9$ & $21.6 \pm 1.4$ & $19.5 \pm 1.2$ \\
\hline $\begin{array}{l}\text { Instantaneous frequency (Hz) } \\
\mathrm{iGC}(n=2)\end{array}$ & $26.7 \pm 2.0$ & $43.1 \pm 2.4$ & $33.0 \pm 2.0$ & $37.5 \pm 2.5$ & $58.2 \pm 2.7$ & $46.4 \pm 2.7$ \\
\hline $\begin{array}{l}\text { \% change (total charge) } \\
\text { RSNP-SOM }\end{array}$ & - & $41.9 \pm 24.2$ & $24.4 \pm 12.3$ & - & $77.6 \pm 47.6$ & $9.1 \pm 11.0$ \\
\hline $\begin{array}{l}\text { Amplitude (pA) } \\
\operatorname{sGC}(n=4)\end{array}$ & $11.8 \pm 0.3$ & $13.5 \pm 0.4$ & $12.1 \pm 0.3$ & $17.1 \pm 0.5$ & $17.6 \pm 0.6$ & $17.2 \pm 0.6$ \\
\hline $\begin{array}{l}\text { Instantaneous frequency }(\mathrm{Hz}) \\
\mathrm{iGC}(n=5)\end{array}$ & $31.3 \pm 1.2$ & $46.1 \pm 2.1$ & $39.1 \pm 2.2$ & $22.2 \pm 1.2$ & $39.0 \pm 1.5$ & $32.4 \pm 1.3$ \\
\hline$\%$ change (total charge) & - & $133.4 \pm 79.2$ & $39.7 \pm 19.8$ & - & $68.0 \pm 9.8$ & $48.4 \pm 29.1$ \\
\hline
\end{tabular}

${ }^{a}$ Average total synaptic charge onto PYR, FS-PV, and RSNP-SOM neurons located in SGC and iGC before, during, and after asynchronous activation of BLA afferents. Data are mean \pm SE.

ceive monosynaptic BLA input (Fig. 3E; Table 1) but can also polysynaptically recruit a significant proportion of the circuit.

\section{Discussion}

The experiments in this study provide a detailed analysis of the connectivity and synaptic properties of the BLA projection to GC. We show that BLA afferents directly target both excitatory and inhibitory neurons and that BLA terminal field stimulation is capable of driving feedforward excitation and feedforward inhibition in aGC. Our data demonstrate that BLA projections contact a larger percentage of neurons in the superficial than in the deep layers of GC and have laminar-specific properties. Furthermore, we show laminar differences in the activation of the superficial and deep layers of aGC under two different activity regimens, using either single stimuli or tonic BLA terminal field activation. Single stimuli of BLA inputs recruited balanced excitation and inhibition in aGC pyramidal neurons, although the amplitude of evoked excitatory and inhibitory synaptic responses

$\leftarrow$

(Figure legend continued.) included in the vertical rectangle. D, CDFs of sPSC amplitude (left) and SEPSC instantaneous frequency (right) in iGC PYR. Black represents baseline. Blue represents ramp. Dashed line indicates after ramp. $\boldsymbol{E}$, Top, Sample trace of spontaneous currents recorded from a FS-PV neuron in SGC (light yellow). Inset, Stretched portion of the trace included in the vertical rectangle. $F$, CDFs of SPSC amplitude (left) and sEPSC instantaneous frequency (right) in sGC FS-PV. Black represents baseline. Light yellow represents ramp. Dashed line indicates after ramp. G, Sample trace of spontaneous currents recorded from a FS-PV neuron in iGC (dark yellow). Inset, Stretched portion of the trace included in the vertical rectangle. $\boldsymbol{H}$, CDFs of sPSC amplitude (left) and sEPSC instantaneous frequency (right) in iGC FS-PV. Black represents baseline. Dark yellow represents ramp. Dashed line indicates after ramp. I, Top, Sample trace of spontaneous currents recorded from a RSNP-SOM neuron in SGC (pink). Inset, Stretched portion of the trace included in the vertical rectangle. J, CDFs of sPSC amplitude (left) and sEPSC instantaneous frequency (right) in SGC RSNP-SOM. Black represents baseline. Pink represents ramp. Dashed line indicates after ramp. $\boldsymbol{K}$, Sample trace of spontaneous currents recorded from a RSNP-SOM neuron in iGC (red). Inset, Stretched portion of the trace included in the vertical rectangle. $L, C D F$ of $S P S C$ amplitude (left) and sEPSC instantaneous frequency (right) in iGC RSNP-SOM Black represents baseline. Red represents ramp. Dashed line indicates after ramp. $\boldsymbol{M}$, Left, Bar plot of percentage change from baseline of total synaptic charge onto PYR in sGC (gray) and iGC (black). Middle, Bar plot of percentage change from baseline of total synaptic charge onto FS-PV in SGC (light yellow) and iGC (dark yellow). Right, Bar plot of percentage change from baseline of total synaptic charge onto RSNP-SOM in SGC (pink) and iGC (red). Left, Ramp. Right, After stimulus. Data are mean \pm SE. *Significant difference (between after stimulus and baseline). Colored asterisk represents significant difference between ramp and baseline. was largest in the superficial layers. Tonic activation of BLA afferents modulated excitatory and inhibitory inputs onto PYR neurons in both layers of aGC. This pattern of stimulation activated superficial layers more intensely than deep layers, a result consistent with the laminar-specific differences in connectivity of the BLA-GC projection. Interestingly, in both layers, inhibition was dominant. Together, these results highlight the wide repertoire of mechanisms by which BLA projections can modulate GC processing.

\section{BLA activates excitatory and inhibitory neurons in aGC}

It is well established that BLA projects to GC and contributes to its processing of hedonic value and taste anticipation (Piette et al., 2012, Samuelsen et al., 2012). However, the specific neuronal targets and the synaptic dynamics of the BLA-GC connection are not well understood. Amygdalocortical afferents are thought to make monosynaptic connections with cortical pyramidal neurons, although recent evidence suggests that they may also drive feedforward inhibition (Stone et al., 2011; Dilgen et al., 2013). Our study demonstrates that the BLA-GC pathway directly activates excitatory and inhibitory neurons. The monosynaptic nature of the BLA-GC connection onto different neuron types is demonstrated by experiments in which spiking activity was blocked by TTX and BLA-EPSCs could still be evoked by increasing the duration of light-induced terminal field depolarization using the potassium channel blocker 4-AP. In addition, we show that the BLA-GC-evoked responses we record are glutamatergic, as AMPA and NMDA receptor blockers completely abolished the monosynaptic current evoked in the presence of TTX and 4-AP. These data, together with our analysis of the proportion of aGC neurons directly activated by BLA afferents stimulation, support the interpretation that excitatory and inhibitory neurons in aGC receive a prominent input from BLA glutamatergic projection neurons.

BLA afferents to aGC engage FS-PV and RSNP-SOM neurons in all layers of GC. These two neuronal populations account for the large majority of inhibitory neurons in cortical circuits (Griffen and Maffei, 2014), suggesting that BLA inputs drive a powerful and widespread activation of feedforward inhibition in aGC. FS-PV inhibitory neurons primarily synapse onto the somatic and perisomatic region of cortical pyramidal neurons, 
A
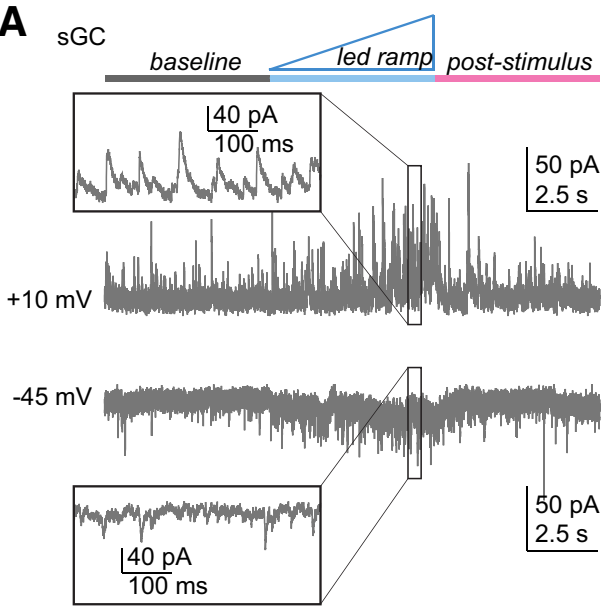

C iGC
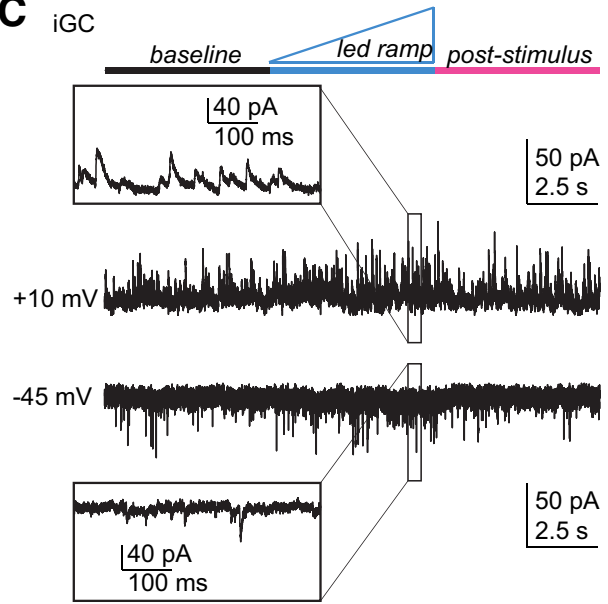

E

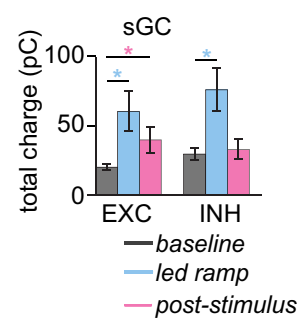

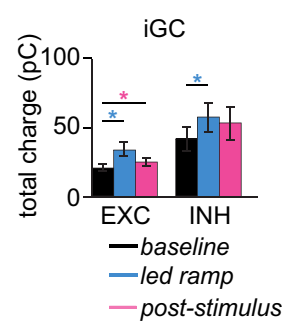

B
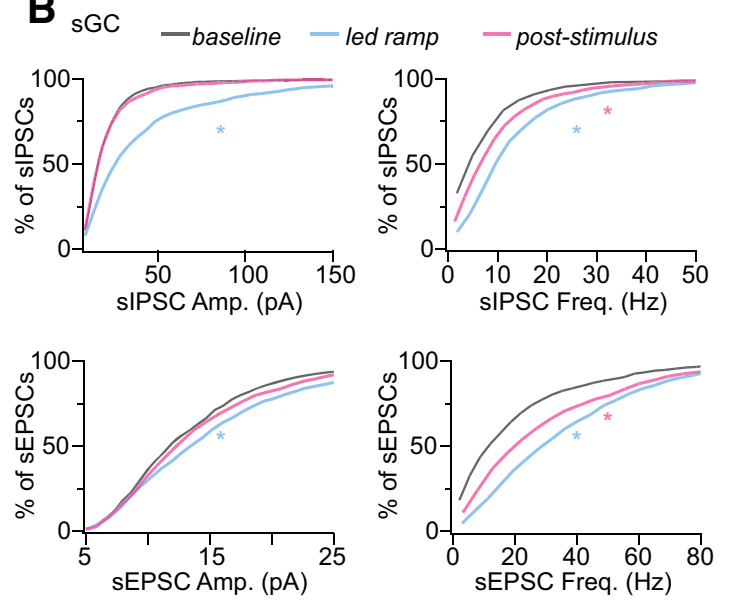

D
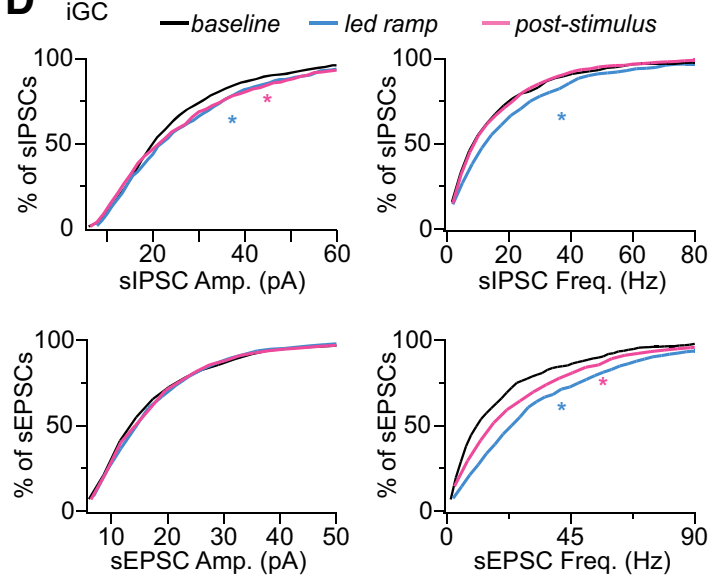

$\mathbf{F}$

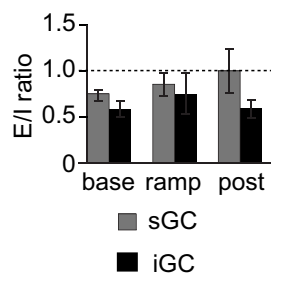

Figure 7. Tonic activation of BLA afferents activates both excitatory and inhibitory circuits in SGC and iGC. $A$, Top, Diagram of experimental design. Light gray represents baseline. Blue represents ramp. Purple represents after ramp. Sample traces of $\mathrm{SGC}$ PYR neuron (gray) held at the reversal potential for excitation (top) and inhibition (bottom) during ramp activation of BLA afferents. Insets, Stretched portion of the trace included in the vertical rectangle. B, Top, CDFs of s IPSC amplitude (left) and sIPSC instantaneous frequency (right) in sGC PYR. Bottom, CDFs of sEPSC amplitude (left) and sEPSC instantaneous frequency (right) in SGC PYR. Light gray represents baseline. Blue represents ramp. Purple represents after ramp. C, Top, Diagram of experimental design. Black represents baseline. Blue represents ramp. Purple represents after ramp. Bottom, Sample traces of iGC PYR neuron (black) held at the reversal potential for excitation (top) and inhibition (bottom) during ramp activation of BLA afferents. Insets, Stretched portion of the trace included in the vertical rectangles. D, Top, CDFs of sIPSC amplitude (left) and IPSC instantaneous frequency (right) in iGC PYR. Bottom, CDFs of sEPSC amplitude (left) and EPSC instantaneous frequency (right) in iGC PYR. Black represents baseline. Blue represents ramp stimulus. Purple represents after ramp. E, Bar plot of total evoked EXC and INH charge onto PYR in SGC (left) and iGC (right). sGC: Gray represents baseline. Light blue represents ramp stimulus. Light purple represents after ramp. iGC: Black represents baseline. Blue represents ramp stimulus. Purple represents after ramp. $\boldsymbol{F}$, E/l ratio of excitatory and inhibitory charge onto PYR in sGC (gray) and iGC (black). $G$, Bar plot of percentage change from baseline of EXC and INH onto PYR in sGC (gray) and iGC (black). Left, Ramp. Right, After stimulus. Data are mean \pm SE. *Significant difference. Blue asterisk represents significant difference between ramp and baseline. Purple asterisk represents significant difference between after stimulus and baseline.

whereas RSNP-SOM preferentially target distal dendritic arbors (Griffen and Maffei, 2014). By driving both populations of inhibitory neurons, BLA inputs can influence somatic and dendritic computations in aGC PYR neurons.

Our analysis of BLA-GC connectivity in superficial and deep layers showed that BLA afferents activate a larger proportion of PYR, FS-PV, and RSNP-SOM neurons in supragranular layers relative to infragranular layers. These results are consistent with the higher density of BLA afferents in the superficial layers of aGC compared with deep layers. In addition, there was a significantly higher amplitude of the light-evoked currents onto PYR neurons in the superficial layers of aGC relative to deep layers. Differently, the amplitude of the responses evoked onto FS-PV and RSNPSOM neurons was similar across layers. Analysis of the shortterm plasticity of BLA synapses activated by trains of light pulses revealed that there are no significant differences in synaptic dynamics either between groups of neurons or across layers. Thus, the laminar specificity of the BLA-GC inputs is primarily depen- 
Table 4. BLA ramp-evoked excitation and inhibition in SGC and iGC ${ }^{a}$

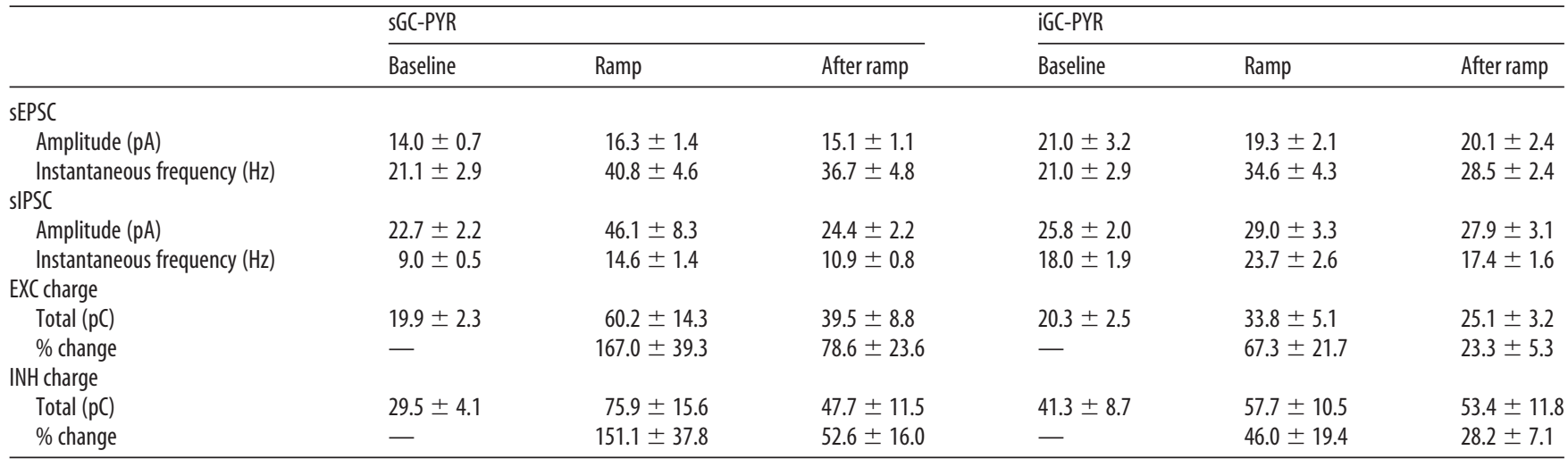

${ }^{a}$ Average amplitude and instantaneous frequency for sEPSCS and sIPSCs as well as total excitatory and inhibitory synaptic charge onto PYR neurons in sGC and iGC before, during, and after asynchronous activation of BLA afferents. Data are mean \pm SE.

A

sGC

$20 \mathrm{pA}$

B
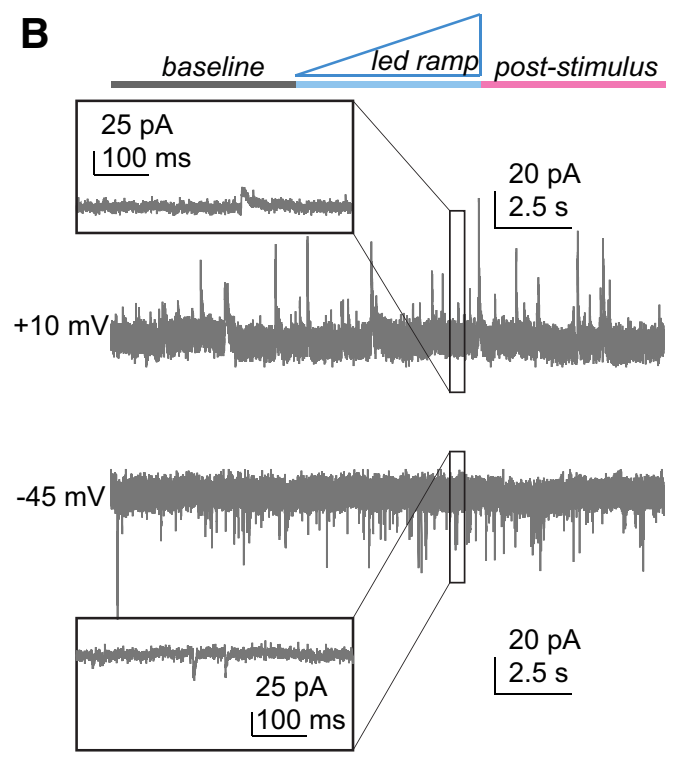

C

IGC

$20 \mathrm{pA}$

$50 \mathrm{~ms}$

D
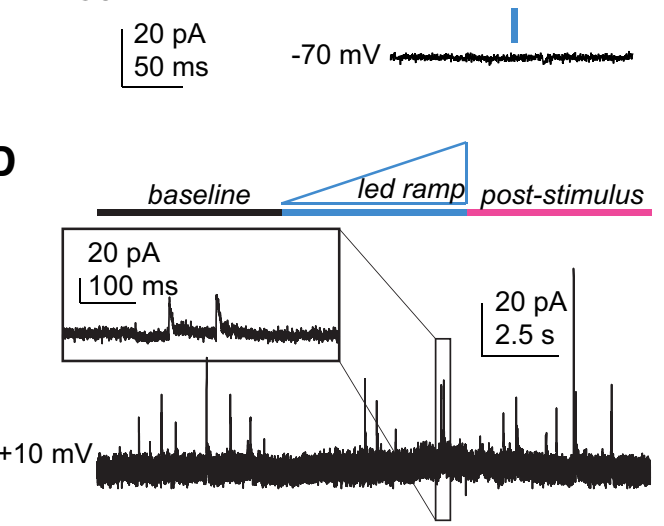

E
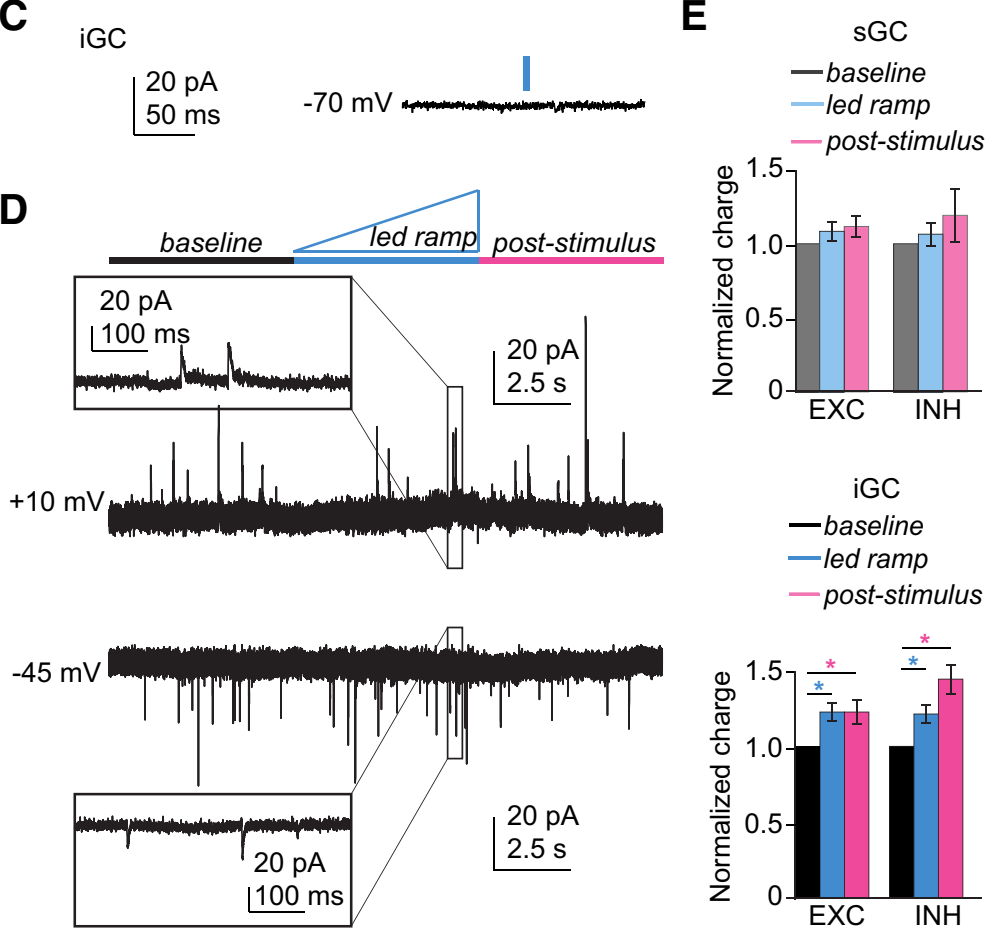

Figure 8. Tonic activation of BLA afferents increases activity in iGC neurons that do not receive direct BLA input. $A$, Sample trace of sGC PYR neuron that does not respond to phasic activation of BLA afferents (gray). Blue rectangle above trace represents the delivery of the light pulse. $\boldsymbol{B}$, Top, Diagram of experimental design. Light gray represents baseline. Blue represents ramp. Purple represents after ramp. Bottom, Sample traces of SGC PYR neuron (gray) that does not receive direct BLA input held at the reversal potential for excitation (top) and inhibition (bottom) during ramp activation of BLA afferents. Insets, Stretched portion of the trace indicated by the vertical rectangles. C, Sample trace of iGC PYR neuron (black) that does not respond to phasic activation of BLA afferents (dark gray). Blue rectangle represents the delivery of the light pulse. D, Top, Diagram of experimental design. Gray represents baseline. Blue represents ramp. Purple represents after ramp. Bottom, Sample trace of iGC PYR neuron (black) that does not receive direct BLA input held at the reversal potential for excitation (top) and inhibition (bottom) during ramp activation of BLA afferents. Insets, Stretched portion of the trace indicated by the vertical rectangles. $E$, Bar plot of total evoked EXC and INH normalized charge onto PYR that do not receive BLA input in sGC (top) and IGC (bottom). sGC: Gray represents baseline. Light blue represents ramp stimulus. Light purple represents after ramp. iGC: Black represents baseline. Blue represents ramp stimulus. Purple represents after ramp. Data are mean $\pm S E$. * Significant difference.

dent on differences in connection probability for all neuron groups and layer-specific BLA-EPSC amplitude selectively onto PYR neurons.

\section{Distinct modes of BLA stimulation differentially recruit the} circuit in aGC

Amygdalar neurons can operate in different activity regimens. Phasic activation of BLA neurons has been associated with the detection of a stimulus and responses to anticipatory cues, whereas tonic activity of BLA neurons is thought to be associated with the valence of a stimulus (Fontanini et al., 2009; Parsana et al., 2012; Samuelsen et al., 2012). In our experiments, we tested how single pulse, multiple pulses, and tonic activation of BLA inputs can recruit feedforward excitatory and inhibitory inputs onto PYR neurons located in the superficial and deep layers of aGC. Our data indicate that single pulse and tonic activation of BLA terminal fields can engage aGC circuits quite differently. Single pulses of light lead to monosynaptic EPSCs and disynaptic IPSCs in PYR neurons. The absolute amplitude of both EPSCs and IPSCs onto pyramidal neurons was larger in the superficial 
than in the deep layers of aGC, consistent with our results showing layer-specific connectivity and amplitude of BLA-evoked responses. The ratio of EPSC and IPSC charge for each PYR neuron was similar in sGC and iGC and was close to 1 . Thus, single BLA stimuli evoked balanced excitation and inhibition in superficial and deep layers of aGC. These results support the interpretation that, although single BLA stimuli drive the superficial layers of aGC more strongly, they activate similar circuit dynamics across all layers of aGC.

Very different circuit dynamics were engaged by tonic activation of BLA afferents. In the superficial and deep layers of aGC, tonic activation of BLA inputs increased the total excitatory and inhibitory charge onto PYR neurons. As for single stimuli, tonic activation of BLA recruited superficial layers more intensely than deep layers. However, for both layers, the ratio of excitatory and inhibitory charge was always $<1$, indicating that this regimen of activation drives the circuit in aGC while maintaining the initial bias toward inhibition. The changes in excitatory and inhibitory activity in neurons directly activated by BLA lasted beyond the end of the ramping stimulus, suggesting that tonic activation of BLA afferents engages aGC recurrent circuitry.

The differences in how excitatory and inhibitory circuits are recruited by single pulses and tonic activity did not depend on neuron-specific or layer-specific short-term synaptic plasticity, as shown by our analysis of BLA-EPSCs in response to trains of light pulses. The lack of differences in short-term plasticity, together with the persistence of activity following the end of the ramp, further supports the interpretation that tonic BLA activation recruits recurrent circuits in sGC and iGC.

Ramping stimuli are thought to engage afferent inputs as well as recurrent cortical circuits (Adesnik and Scanziani, 2010). In our experimental setting, we could test this by assessing whether BLA stimulation could modulate the activity of PYR neurons that do not receive direct BLA input. This approach allowed us to further identify laminar differences between the superficial and deep layers of aGC. In sGC, tonic BLA activation did not affect excitatory and inhibitory inputs onto PYR neurons that were not directly activated by BLA stimulation, suggesting that, in sGC, neurons that receive direct input from BLA may be part of a distinct functional circuit. It should be noted that BLA afferents in sGC drive the majority of excitatory and inhibitory neurons monosynaptically; thus, BLA inputs have a direct and widespread effect on the circuit in sGC.

Differently, BLA afferents directly contact only a small proportion of iGC neurons, yet tonic activation of BLA afferents increased both excitatory and inhibitory charge even onto a fairly large population of neurons that do not receive direct BLA inputs. These results suggest that, whereas BLA connectivity in iGC is much less extensive than in SGC, tonic BLA activity can recruit a significant portion of the iGC circuit via polysysnaptic interactions.

\section{Functional implications}

Neurons in distinct layers of sensory cortices other than GC have been shown to encode information differently (Petersen and Crochet et al., 2013). The laminar processing of sensory stimuli depends on many factors, such as differences in thalamic inputs, local connectivity, specific properties of cortical neurons, release of neuromodulators, and distribution of long range projections. Our experimental results show significant laminar differences in BLA-GC connectivity and synaptic properties, suggesting that neurons in different layers of aGC may respond with laminarspecific dynamics to inputs carrying information about anticipatory cues or the hedonic value of a stimulus. Although evidence for layer specificity of taste processing in GC of alert animals is currently missing, future studies will need to address this issue, specifically in relation to amygdalar inputs.

In addition, our data show that BLA activity regimens known to be relevant for behavior can differentially engage excitatory and inhibitory circuits. The balance between excitation and inhibition within the circuit differs significantly in response to single pulses and tonic activation. Single action potentials or phasic bursts of activity from BLA, typically occurring in response to anticipatory cues or onset of taste delivery (Fontanini et al., 2009; Samuelsen et al., 2012), may result in strong and balanced inputs to GC. These inputs might be responsible for driving GC neurons to fire. On the contrary, tonic BLA activity, characteristic of persistent responses to taste associated with palatability (Fontanini et al., 2009; Piette et al., 2012), may lead to an increase of inhibitory tone in GC. These pattern-specific changes in E/I balance suggest that inhibition plays an important role in modulating GC processing of different variables associated with a gustatory experience.

\section{References}

Adachi K, Fujita S, Yoshida A, Sakagami H, Koshikawa N, Kobayashi M (2013) Anatomical and electrophysiological mechanisms for asymmetrical excitatory propagation in the rat insular cortex: in vivo optical imaging and whole-cell patch-clamp studies. J Comp Neurol 521:1598-1613. CrossRef Medline

Adesnik H, Scanziani M (2010) Lateral competition for cortical space by layerspecific horizontal circuits. Nature 464:1155-1160. CrossRef Medline

Allen GV, Saper CB, Hurley KM, Cechetto DF (1991) Organization of visceral and limbic connections in the insular cortex of the rat. J Comp Neurol 311:1-16. CrossRef Medline

Balleine BW, Killcross S (2006) Parallel incentive processing: an integrated view of amygdala function. Trends Neurosci 29:272-279. CrossRef Medline

Baxter MG, Murray EA (2002) The amygdala and reward. Nat Rev Neurosci 3:563-573. CrossRef Medline

Chavez CM, McGaugh JL, Weinberger NM (2009) The basolateral amygdala modulates specific sensory memory representations in the cerebral cortex. Neurobiol Learn Mem 91:382-392. CrossRef Medline

Cruikshank SJ, Urabe H, Nurmikko AV, Connors BW (2010) Pathwayspecific feedforward circuits between thalamus and neocortex revealed by selective optical stimulation of axons. Neuron 65:230-245. CrossRef Medline

Dilgen J, Tejeda HA, O’Donnell P (2013) Amygdala inputs drive feedforward inhibition in the medial prefrontal cortex. J Neurophysiol 110:221229. CrossRef Medline

Faber ES, Sah P (2002) Physiological role of calcium-activated potassium currents in the rat lateral amygdala. J Neurosci 22:1618-1628. Medline

Ferreira G, Miranda MI, De la Cruz V, Rodríguez-Ortiz CJ, BermúdezRattoni F (2005) Basolateral amygdala glutamatergic activation enhances taste aversion through NMDA receptor activation in the insular cortex. Eur J Neurosci 22:2596-2604. CrossRef Medline

Fontanini A, Katz DB (2006) State-dependent modulation of time-varying gustatory responses. J Neurophysiol 96:3183-3193. CrossRef Medline

Fontanini A, Grossman SE, Figueroa JA, Katz DB (2009) Distinct subtypes of basolateral amygdala taste neurons reflect palatability and reward. J Neurosci 29:2486-2495. CrossRef Medline

Gardner MP, Fontanini A (2014) Encoding and tracking of outcomespecific expectancy in the gustatory cortex of alert rats. J Neurosci 34: 13000-13017. CrossRef Medline

Griffen TC, Maffei A (2014) GABAergic synapses: their plasticity and role in sensory cortex. Front Cell Neurosci 8:91. CrossRef Medline

Grossman SE, Fontanini A, Wieskopf JS, Katz DB (2008) Learning-related plasticity of temporal coding in simultaneously recorded amygdalacortical ensembles. J Neurosci 28:2864-2873. CrossRef Medline

Hanamori T (2009) Effects of electrical and chemical stimulation of the amygdala on the spontaneous discharge in the insular cortex in rats. Brain Res 1276:91-102. CrossRef Medline

Jackman SL, Beneduce BM, Drew IR, Regehr WG (2014) Achieving highfrequency optical control of synaptic transmission. J Neurosci 34:7704-7714. CrossRef Medline 
Lin JY, Lin MZ, Steinbach P, Tsien RY (2009) Characterization of engineered channelrhodopsin variants with improved properties and kinetics. Biophys J 96:1803-1814. CrossRef Medline

Maffei A, Haley M, Fontanini A (2012) Neural processing of gustatory information in insular circuits. Curr Opin Neurobiol 22:709-716. CrossRef Medline

ParéD, Smith Y, ParéJF (1995) Intra-amygdaloid projections of the basolateral and basomedial nuclei in the cat: phaseolus vulgaris-leucoagglutinin anterograde tracing at the light and electron microscopic level. Neuroscience 69:567-583. CrossRef Medline

Parsana AJ, Li N, Brown TH (2012) Positive and negative ultrasonic signals elicit opposing firing patterns in rat amygdala. Behav Brain Res 226: 77-86. CrossRef Medline

Pessoa L, Adolphs R (2010) Emotion processing and the amygdala: from a 'low road' to 'many roads' of evaluating biological significance. Nat Rev Neurosci 11:773-783. CrossRef Medline

Petersen CC, Crochet S (2013) Synaptic computation and sensory processing in neocortical layer 2/3. Neuron 78:28-48. CrossRef Medline

Petreanu L, Huber D, Sobczyk A, Svoboda K (2007) Channelrhodopsin-2assisted circuit mapping of long-range callosal projections. Nat Neurosci 10:663-668. CrossRef Medline

Phelps EA, LeDoux JE (2005) Contributions of the amygdala to emotion processing: from animal models to human behavior. Neuron 48:175-187. CrossRef Medline

Piette CE, Baez-Santiago MA, Reid EE, Katz DB, Moran A (2012) Inactivation of basolateral amygdala specifically eliminates palatability-related information in cortical sensory responses. J Neurosci 32:9981-9991. CrossRef Medline
Rodríguez-Durán LF, Castillo DV, Moguel-González M, Escobar ML (2011) Conditioned taste aversion modifies persistently the subsequent induction of neocortical long-term potentiation in vivo. Neurobiol Learn Mem 95:519-526. CrossRef Medline

Roesch MR, Calu DJ, Esber GR, Schoenbaum G (2010) Neural correlates of variations in event processing during learning in basolateral amygdala. J Neurosci 30:2464-2471. CrossRef Medline

Samuelsen CL, Gardner MP, Fontanini A (2012) Effects of cue-triggered expectation on cortical processing of taste. Neuron 74:410-422. CrossRef Medline

Samuelsen CL, Gardner MP, Fontanini A (2013) Thalamic contribution to cortical processing of taste and expectation. J Neurosci 33:1815-1827. CrossRef Medline

Saper CB (1982) Convergence of autonomic and limbic connections in the insular cortex of the rat. J Comp Neurol 210:163-173. CrossRef Medline

Smith Y, Paré D (1994) Intra-amygdaloid projections of the lateral nucleus in the cat: PHA-L anterograde labeling combined with postembedding GABA and glutamate immunocytochemistry. J Comp Neurol 342: 232-348. CrossRef Medline

Stone ME, Maffei A, Fontanini A (2011) Amygdala stimulation evokes time-varying synaptic responses in the gustatory cortex of anesthetized rats. Front Integr Neurosci 5:3. CrossRef Medline

Wang L, Kloc M, Gu Y, Ge S, Maffei A (2013) Layer-specific experiencedependent rewiring of thalamocortical circuits. J Neurosci 33:4181-4191. CrossRef Medline

Yamamoto T, Azuma S, Kawamura Y (1984) Functional relations between the cortical gustatory area and the amygdala: electrophysiological and behavioral studies in rats. Exp Brain Res 56:23-31. Medline 